\title{
Structure of resonances and formation of stationary points in symmetrical chains of bilinear oscillators
}

\author{
Arcady V. Dyskin ${ }^{1}$, Elena Pasternak ${ }^{2}$, Igor Shufrin ${ }^{1,2}$ \\ ${ }^{1}$ School of Civil and Resource Engineering, The University of Western Australia, 35 Stirling \\ Highway, CrawleyWA 6009, Australia, Email: arcady.dyskin@uwa.edu.au \\ ${ }^{2}$ School of Mechanical and Chemical Engineering, The University of Western Australia, 35 \\ Stirling Highway, Crawley WA 6009, Australia
}

\begin{abstract}
Dynamics of strongly non-linear systems can in many cases be modelled by bilinear oscillators, which are the oscillators whose springs have different stiffnesses in compression and tension. This underpins the analysis of a wide range of phenomena, from oscillations of fragmented structures, connections and mooring lines to deformation of geological media. Single bilinear oscillators were studied previously and the presence of multiple resonances both super- and sub-harmonic was found. Less attention was paid to systems of multiple bilinear oscillators that describe many natural and engineering processes such as for example the behaviour of fragmented solids. Here we fill this gap concentrating on the simplest case - 1D symmetrical chains of bilinear oscillators. We show that the presence and structure of resonances in a symmetric chain of bilinear oscillators with fixed ends depends upon the number of oscillating masses. Two elementary chains act as the basic ones: a single mass bilinear chain (a mass connected to the fixed points by two bilinear springs) that behaves as a linear oscillator with a single resonance and a two mass chain that is a coupled bilinear oscillator (two masses connected by three bilinear springs). The latter has multiple resonances. We demonstrate that longer chains either do not have resonances or get decomposed, during the resonance, into either the single mass or two mass elementary chains with stationary masses in between. The resonance frequencies are inherited from the basic chains of decomposition. We show that if the number of masses is odd the chain can be decomposed into the single mass bilinear chains separated by stationary masses. It then inherits the resonances of the single mass bilinear chain. The chains with the number of masses minus 2 divisible by 3 can be decomposed into the two mass bilinear chains separated by stationary masses and inherit the resonances of the two mass chains. The chains whose lengths satisfy both criteria (such as chains with $5,11,17 \ldots$ masses) allow both types of resonances.
\end{abstract}


Keywords: Bilinear spring, Linear states, Resonances, Stationary points, Resonant decomposition

\section{Introduction}

The bilinear oscillator, an oscillator consisting of a mass and a spring with different stiffnesses in tension and compression is the simplest model of highly non-linear systems with returning forces strongly depending upon the direction of movement. The systems of this kind can be encountered in cutting as the movement of the cutting tool towards the workpiece creates high contact forces, while the movement away from it meets a lower resistance for the tool holder. The difference is especially high in borehole drilling where the role of the holder is played by a drill string, which is long and hence has low longitudinal stiffness. This may cause intermittent engagement between the workpiece and the tool and induce strong vibrations limiting the material removal rates, especially at high speeds (e.g., [1-3]). Other examples include mooring lines and towers (e.g., [47]), the dynamics of structures with gaps and contacts such as suspension bridges [8-9], connections of carriages in trains [10], thin cracks in engineering and geo-materials [11-22], as well as biological systems [23]. In addition, bilinear oscillator models are employed in the mechanics of rigid body impacts [24-27] and modelling of friction induced discontinuities [28]. A recent example is provided by topological interlocking structures [29], where the elements are held together via kinematic constraints rather than a binder or connectors. Topological interlocking structures exhibit very high sound absorption [30], which diminishes as soon as the interlocking blocks are glued together. This interesting feature is attributed to the bilinear nature of contact interfaces between the interlocked blocks. Furthermore, since blocks in the topological interlocking structures cannot move independently, they form a system of coupled bilinear oscillators generating intricate dynamic behaviour.

A bilinear oscillator is described by the following equation

$$
M \ddot{x}+b \dot{x}+k(x) x=f(t), k(x)=\left\{\begin{array}{cc}
k_{+}, & x \geq 0 \\
k_{-}, & x<0
\end{array},\right.
$$

Here $M$ is the mass of the oscillator, $x(t)$ is the trajectory of the oscillator, $b$ is the damping coefficient and $k(x)$ is the direction dependent bilinear stiffness. For the sake of definiteness, we assume that $k_{+}<k_{-}$. We note that given the driving force $f(t)$ is continuously differentiable, 
solution of Eqn. (1) is twice continuously differentiable while its third derivative is discontinuous at $x=0$.

The phase space of the bilinear oscillator is split into two linear states, while the non-linearity is associated with crossing the boundary between them. The resonant frequency can be obtained by summing up the times oscillator spent in each of the linear states; the result is (e.g., [4, 31])

$$
\Omega_{r}=\frac{2 \Omega_{+} \Omega_{-}}{\Omega_{+}+\Omega_{-}}, \quad \Omega_{ \pm}^{2}=\omega_{ \pm}^{2}-\alpha^{2}, \quad \omega_{ \pm}^{2}=\frac{k_{ \pm}}{M}, \quad \alpha=\frac{b}{M}
$$

An important feature of a bilinear oscillator is the presence of super-harmonic and sub-harmonic resonances with respect to $\Omega_{r}$ (e.g. [4, 31]). Resonances of a single degree-of-freedom bilinear oscillator at frequencies that are multiple and fractional to the fundamental one were analysed in [32-35]. The sub-harmonic resonances, i.e. resonances excited on fractional frequencies are different from the conventional resonances in that (in the absence of damping) the amplitude of the oscillations increases slower than linear. For instance for a resonance at a half eigenfrequency the amplitude increases as a square root of time [35]. The super-harmonic and sub-harmonic resonances were also experimentally detected in materials with cracks and defects [36, 37].

In many cases engineering and natural systems are described by systems of bilinear oscillators. These include the trains, the structures of interlocking blocks and generally mortarless structures. The natural systems whose oscillations can be modelled by systems of bilinear oscillators include granular media as well as heavily fractured and blocky rock masses. It is important to describe the resonances of such structures since oscillations at a resonance frequency can be detrimental to their stability. In particular, the presence of super- and sub-harmonic resonances associated with the bilinear oscillators makes these systems excitable considerably easier than the systems of linear oscillators. Here we investigate the structure of resonances of a one-dimensional system of coupled bilinear oscillators.

The systems of bilinear oscillators are characterised by the presence of multiple linear states such that the non-linearity is localised in the transition points between the states [29]. Therefore computationally, any trajectory is a concatenation of linear solution with special transition points 
between them. This consideration was a basis for the analysis of a system of two identical masses connected to each other and to the fixed boundaries by identical bilinear springs, Figure 1. It was found that the main resonance could only be achieved in a motion that alternates linear states with identical eigenvectors. The resonance frequency is determined from the eigenfrequencies corresponding to these eigenvectors using Eqn. (2); this model will be reproduced in detail in the following section.

A chain of $n$ impact oscillators fixed at one end and free at another was considered in [38]. The energy loss at the impact was characterised by the coefficient of restitution. For two oscillators $(n=2)$ a periodic sticking motion was found. A coupled bilinear oscillator with a free end was considered in $[39,40]$. The analysis was based on combining the eigenfrequencies from alternating linear states, however out of 4 possibilities only two were considered in the approximation presented. A periodic solution for a more general system of bilinear oscillators was reported in [41]. This method was based on the classical perturbation procedure, while the matching of motions from different linear states was done by non-smooth temporal transformation. Twodegree-of-freedom system similar to the one shown in Figure 1 but with two linear springs and one bilinear spring was used as the example.

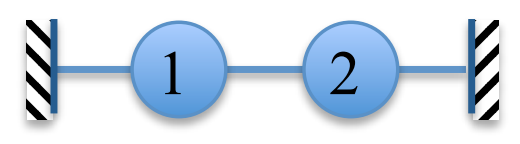

Figure 1. A two mass elementary bilinear chain - a coupled bilinear oscillator. The lines represent identical bilinear springs. The masses are assumed to have only longitudinal movements.

Essentially, the generalisation to higher numbers of oscillators is complicated by the fact that the number of linear states is $2^{n+1}$, where $n$ is the number of masses, $n+1$ is the number of bilinear springs. This intrinsic exponential complexity of the problem calls for developing different methods of analysis. In this paper, we propose such a method for a closed chain of identical bilinear oscillators without damping, Figure 2. Using this method that capitalises on the symmetry of the chain, we will demonstrate that during resonances the chain is decomposed into elementary 
subsystems, Figure 1 and 3 with stationary masses in between, as was found in [42] through numerical experiments. For the sake of simplicity, we will consider oscillators without damping. We also assume that (a) all the masses are equal and the variables are normalised in such a way that $M=1$ and (b) the bilinear stiffnesses specified in Eqn. (1) are the same for all springs.

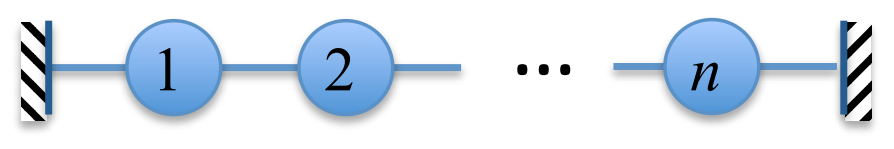

Figure 2. Closed chain of $n$ bilinear oscillators (the links between the masses and between the masses and the rests are identical bilinear springs). The masses are assumed to have only longitudinal movements.

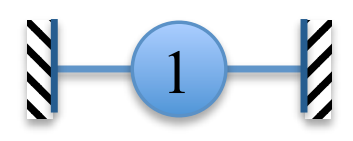

Figure 3. A single mass bilinear chain - a mass on two identical bilinear springs. It behaves as a conventional linear oscillator.

The structure of this paper is as follows: Section 2 describes the structure of resonances of single mass and two mass bilinear chains, which are the basic oscillators of the chain, Section 3 presents the symmetry considerations and describes the resonance decompositions, Section 4 presents numerical examples and Section 5 discusses possible applications of the findings.

\section{Two elementary bilinear chains}

We start with considering two basic oscillators which correspond to the chain with $n=1$ (Figure 3) and $n=2$ (Figure 1). The single mass bilinear chain, Figure 3, is effectively a linear oscillator since stretching one spring leads to contraction of another. The masses are assumed to have only longitudinal movements. Therefore it always has the same stiffness:

$$
k=k_{+}+k_{-}
$$

Given normalisation $M=1$ the resonance frequency of the oscillator is 


$$
\Omega_{1}=\sqrt{k_{+}+k_{-}}
$$

The two mass bilinear chain, Figure 1, can be analysed by considering the linear states and transitions between them [29]. To this end, consider a two DOF system with two identical masses and three identical bilinear springs, Figure 2. The free vibrations of this system are described by the following system of equations:

$$
\left\{\begin{array}{l}
\ddot{x}_{1} \\
\ddot{x}_{2}
\end{array}\right\}+\left[\begin{array}{cc}
k\left(x_{1}\right)+k\left(x_{2}-x_{1}\right) & -k\left(x_{2}-x_{1}\right) \\
-k\left(x_{2}-x_{1}\right) & k\left(x_{2}-x_{1}\right)+k\left(-x_{2}\right)
\end{array}\right]\left\{\begin{array}{l}
x_{1} \\
x_{2}
\end{array}\right\}=\left\{\begin{array}{l}
0 \\
0
\end{array}\right\}, k(x)=\left\{\begin{array}{cc}
k_{+}, & x \geq 0 \\
k_{-}, & x<0
\end{array}\right.
$$

where $x_{1}$ and $x_{2}$ are the positions of masses 1 and 2 respectively.

This system has 8 possible combinations of the spring stiffnesses (states), however only 6 of them are physically admissible as in this one dimensional chain all the springs cannot be simultaneously in compression or in tension, since only longitudinal oscillations are possible. Accordingly, the transition points between the states are at $x_{1}=0, x_{2}=0$, and $x_{1}=x_{2}$. We designate the states by the sense of the springs from left to right. For example, $(+,+,-)$ denotes a state in which the first and the second springs are in tension and the third one is in compression. For each combination of the stiffnesses, Eqns. (5) represents a linear system of equations whose solution yields two eigenfrequencies and two corresponding eigenvectors. These are listed in Table 1 for all linear states, where $K$ is the non-linearity factor defined as

$$
K=\frac{k_{-}}{k_{+}}=\left(\frac{\omega_{-}}{\omega_{+}}\right)^{2}
$$

A resonance involves alternation of two eigenmodes from different linear states. The resonance frequency is then composed of the frequencies of these modes, using Eqn. (2) with $\alpha=0, M=1$ as

$$
\Omega_{R}=2 \frac{\omega_{i} \omega_{j}}{\omega_{i}+\omega_{j}}
$$

where $\omega_{i}$ and $\omega_{j}$ are the eigenfrequencies of the modes involved into the transition. 
A resonance at this frequency involves the motion that alternates between the states $i$ and $j$ ensuring the multiple transitions from the state $i$ to the state $j$ and vice versa. This is only possible when the eigenvectors corresponding to $\omega_{i}$ and $\omega_{j}$ are identical. Thus according to Table 1 , the only available resonance transition is between states 3 and 6, whose pairs of eigenvectors coincide. However, out of two eigenvectors from states 3 and 6 only one can sustain resonance. Figure 4 illustrates the motions that correspond to eigenvectors of states 3 and 6 . It is seen that in the case of vector $(-1,10$ the masses move in opposite directions (Figure $4 \mathrm{a})$, while in the case of vector $(1,1)$ they move in the same direction (Figure 4b). The equal movement of both masses in the same direction means that the spring in the middle should stay neutral, neither in tension nor in compression. Obviously, this state cannot maintain itself, as an infinitesimal disturbance will throw the spring into either tensile or compressive state. Indeed, in terms of displacement the spring displacement $u$ the neutral state consists one point, $u=0$. This one point set cannot sustain an infinitesimal change in displacement. On the other hand, the tensile $(u>0)$ or compressive $(u<0)$ states are represented by open sets, which allow infinitesimal change in displacement (for every, say $u>0$, there exist $\Delta u$ such that $u+\Delta u>0$ ). This circumstance will drive the system out of states 3 or 6 needed for the resonance that is why the resonance is not possible for the eigenvector $(1,1)$.

Table 1. Eigensolutions of the linear states of a two mass bilinear chain - a two degree-of-freedom bilinear oscillator

\begin{tabular}{|c|c|c|c|}
\hline $\begin{array}{l}\text { State } \\
\text { No }\end{array}$ & $\begin{array}{l}\text { State } \\
\text { Designation }\end{array}$ & Eigenvectors & Eigenfrequencies \\
\hline 1 & $(+,+,+)$ & Impossib & \\
\hline 2 & $(+,+,-)$ & $\left.1, \quad \frac{1}{2}\left(1-K \pm \sqrt{(K-1)^{2}+4}\right)\right)$ & $\omega_{+} \sqrt{\frac{1}{2}\left(K+3 \mp \sqrt{(K-1)^{2}+4}\right)}$ \\
\hline 3 & $(+,-,+)$ & $\mp 1, \quad 1)$ & $\omega_{+} \sqrt{1+K \pm K}$ \\
\hline 4 & $(+,-,-)$ & $1, \quad \frac{1}{2 K}\left(1-K \pm \sqrt{(K-1)^{2}+4 K^{2}}\right)$ & $\omega_{+} \sqrt{\frac{1}{2}\left(3 K+1 \mp \sqrt{(K-1)^{2}+4 K^{2}}\right.}$ \\
\hline 5 & $(-,+,+)$ & $1, \quad \frac{1}{2}\left(K-1 \pm \sqrt{(K-1)^{2}+4}\right)$ & $\omega_{+} \sqrt{\frac{1}{2}\left(K+3 \mp \sqrt{(K-1)^{2}+4}\right)}$ \\
\hline 6 & $(-,+,-)$ & $\mp 1,1)$ & $\omega_{+} \sqrt{1+K \pm 1}$ \\
\hline 7 & $(-,-,+)$ & $1, \frac{1}{2 K}\left(K-1 \pm \sqrt{(K-1)^{2}+4 K^{2}}\right)$ & $\omega_{+} \sqrt{\frac{1}{2}\left(3 K+1 \mp \sqrt{(K-1)^{2}+4 K^{2}}\right.}$ \\
\hline 8 & $(-,-,-)$ & Impossib & \\
\hline
\end{tabular}




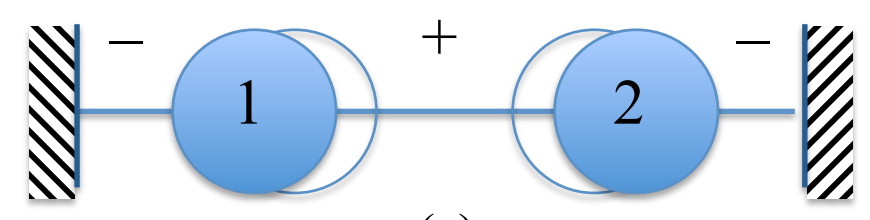

(a)

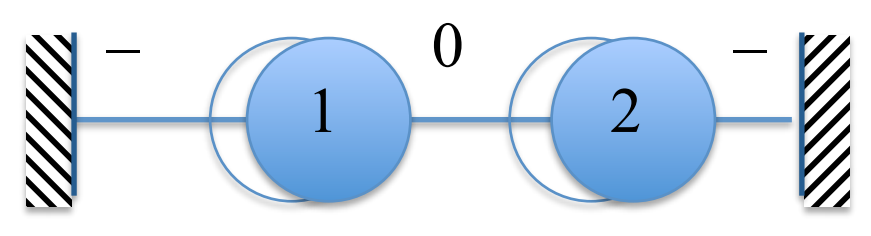

(b)

Figure 4. Eigenmodes of states 3 and 6 of the two mass bilinear chain: (a) eigenvector $(-1,1)$, all springs alternate their tensile (marked as + ) and compressive (marked as -) states; (b) eigenvector $(1,1)$, the central spring reminds neutral (marked as 0 ) through the whole process of oscillations.

Open circles mark the initial positions of the masses.

As a result, we only have a single combination of the eigenmodes complying with all restrictions: the first mode $(-1,1)$ from states 3 and 6 . Thus, the resonant frequency determined from the frequencies of these modes is:

$$
\Omega_{R}=2 \frac{\sqrt{1+2 K} \sqrt{2+K}}{\sqrt{1+2 K}+\sqrt{2+K}} \omega_{+}
$$

This is the (only) resonance frequency found in [29] by numerical simulation.

\section{Symmetries of closed chains of bilinear oscillators and resonant decomposition}

Using the reasoning from the previous section for closed chains with larger numbers of masses (Figure 2) is technically difficult as the number of linear states growth exponentially with the number of masses. Instead, we employ the symmetry considerations to simplify the analysis. 
Suppose $\mathbf{x}(t)=\left(x_{1}(t), \ldots, x_{n}(t)\right)$ is a trajectory of the symmetric closed chain of bilinear oscillators under some general (arbitrary) initial conditions, where $x_{p}(t), p=1, \ldots, n$ is displacement of mass $p$. The trajectories of the chain, Figure 2 are governed by the following system of differential equations

$$
\left\{\begin{array}{l}
\ddot{x}_{p}+k\left(x_{p}-x_{p-1}\right)\left(x_{p}-x_{p-1}\right)-k\left(x_{p+1}-x_{p}\right)\left(x_{p+1}-x_{p}\right)=0, \quad p=1, \ldots, n \\
x_{p}(0)=x_{p}^{0} \\
\dot{x}_{p}(0)=x_{p}^{1}
\end{array}\right.
$$

For the sake of completeness, we add the fixed boundary conditions $x_{0}=x_{n+1}=0$.

Introduce, following [43], symmetrical transformations, which are linear maps $\gamma: \mathbf{R}^{n} \rightarrow \mathbf{R}^{n}$ that send solutions of the system of differential equations (9) to solutions. In particular we consider the following transformations

$$
\gamma_{k}:\left(x_{1}, \ldots x_{k}, \ldots x_{n+1-k}, \ldots x_{n}\right) \mapsto\left(x_{1}, \ldots x_{n+1-k}, \ldots x_{k}, \ldots x_{n}\right)
$$

Due to symmetry, these transformations map trajectories into trajectories: $\gamma_{k} \mathbf{x}(t)=\mathbf{x}\left(t+\theta_{k}\right)$. Furthermore, if the trajectory is periodic with a period $T$, then because $\gamma_{k}^{2}=1$, where 1 is the identity, either $2 \theta_{k}=0$ or $2 \theta_{k}=T$. From here one has $\theta_{k}=0$ or $T / 2$.

Following the reasoning from the previous section we only consider what we will refer to as stable trajectories (motion). These are the trajectories, in which a spring does not stay in a neutral position (neither tension nor compression) during a time interval. Occupation of a neutral position over a finite time interval is obviously not sustainable), since any random infinitesimal disturbance will cause the system to fall unpredictably into one of the states (see an explanation at the end of previous section.

Next we consider two separate cases of odd and even numbers of masses. 


\subsection{Odd number of masses}

Consider the case of odd number of masses, $n=2 m+1$, Figure 5. Apply transformation $\gamma_{1}=\gamma_{1} \gamma_{2} \cdots \gamma_{m}$. This is a transformation that numbers the masses in a reverse order. Two cases are possible: $\gamma \mathbf{x}(t)=\mathbf{x}(t)$ and $\gamma \mathbf{x}(t)=\mathbf{x}(t+T / 2)$.

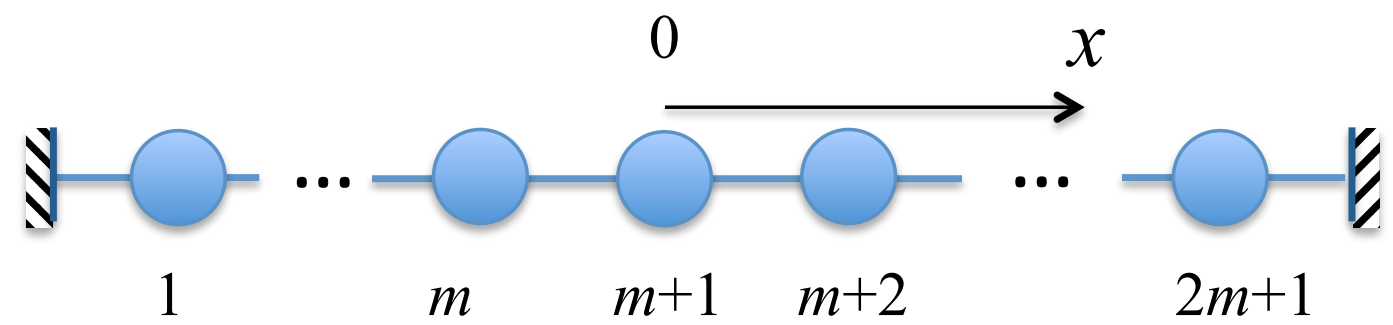

Figure 5. Odd number of masses in the chain

Case 1: $\gamma \mathbf{x}(t)=\mathbf{x}(t)$.

In this case, for the central mass one has $\gamma x_{m+1}(t)=x_{m+1}(t)$. On the other hand, the same transformation is equivalent to sending the $x$-axis in the opposite direction, i.e. to coordinate transformation $x \mapsto-x$. This implies that $\gamma x_{m+1}(t)=-x_{m+1}(t)$. From here we infer that $x_{m+1}(t) \equiv 0$. This is a stationary point in the chain. Therefore, the periodic oscillations of a system of $2 m+1$ masses are equivalent to oscillations of 2 independent systems of $m$ masses, Figure 6a. Thus, a long system is decomposed into two shorter decoupled systems. This constitutes a step of mathematical induction, which is used later on. 


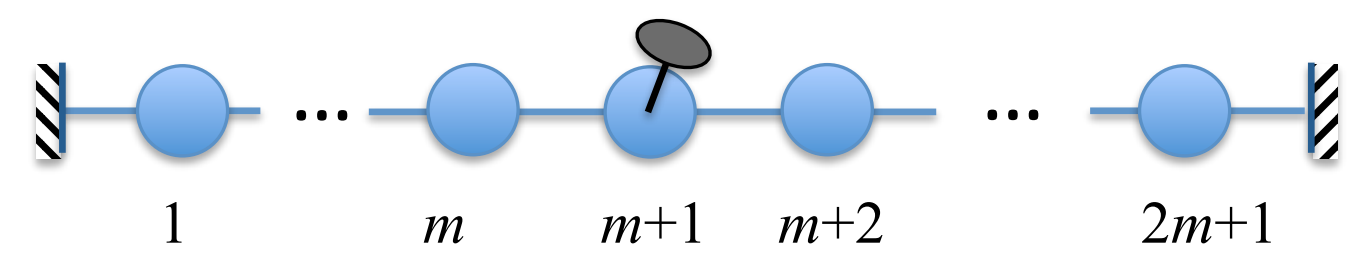

(a)

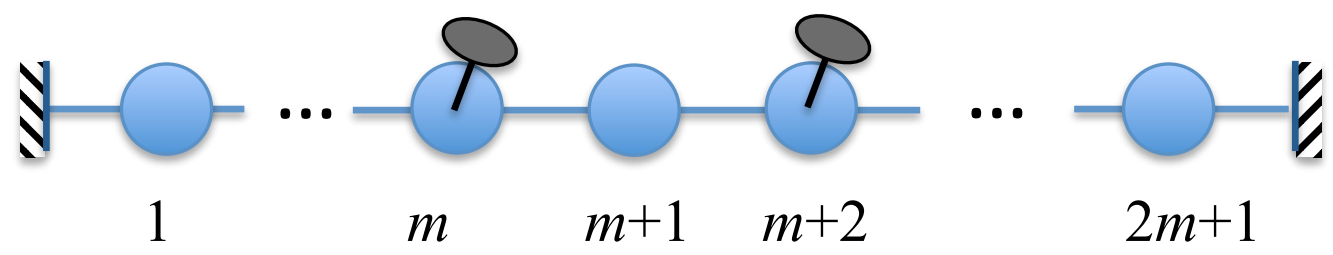

(b)

Figure 6. Two possibilities of splitting the system of $2 m+1$ masses into smaller independent subsystems: (a) splitting into two systems of $m$ masses by making the central mass stationary (this is indicated by a nail driven into the central mass); (b) splitting into three systems - two systems of $m-1$ masses and a single mass system at the centre. The 'nails' in the picture mark the masses that remain stationary in splitting the system into smaller ones.

Case 2: $\gamma \mathbf{x}(t)=\mathbf{x}(t+T / 2)$.

Applying the same coordinate transformation as in the previous case $x \mapsto-x$, we obtain that $-\mathbf{x}(t)=\mathbf{x}(t+T / 2)$.

Consider the system of differential equations (10) that governs the chain. We can rewrite (10) as

$$
\ddot{x}_{p}+\left[k\left(x_{p}-x_{p-1}\right)+k\left(x_{p+1}-x_{p}\right)\right] x_{p}-k\left(x_{p}-x_{p-1}\right) x_{p-1}-k\left(x_{p+1}-x_{p}\right) x_{p+1}=0, p=1, \ldots, n
$$

Suppose at time $t$ the system is in the linear state $S^{1}$. At time $t+T / 2$, the system either stays in $S^{1}$ or moves to another linear state, call it $S^{2}$. (If the system is still in $S^{1}$, the time the system stays in the state that follows $S^{1}$ is shorter than $T / 2$; we will then start with that state and call it $S^{1}$. In other words, $S^{1}$ is always the state such that the time the system stays there is shorter than $T / 2$.) 
Now we only have to consider the situation when at time $t+T / 2$ the system has moved to state $S^{2}$. Then because of $\mathbf{x}(t+T / 2)=-\mathbf{x}(t)$ the system has the same trajectory as in $S^{1}$, but with the sign reversed, at least in an interval of $t$, which includes the time when the system started in $S^{1}$. As the system of Eqns. (11) is homogeneous, the change of sign in $\mathbf{x}$ does not change the equations as long as the stiffnesses are unchanged.

We conclude from the reasoning of the previous paragraph that the system of equations should have the same stiffnesses in both states. Indeed, after changing the sign of the trajectory in $S^{2}$ we will have the situation when the systems of equations describing both states have the same solution, $\mathbf{x}(t) \neq 0$. Then the difference between them is a system of homogeneous algebraic equations $A \mathbf{x}(t)=0$, where $A$ is a constant matrix whose elements are the differences between the stiffnesses in states $S^{1}$ and $S^{2}$. If the rank of matrix $A, r>0$ then there are $n-r$ independent components of $\mathbf{x}(t)$. It follows that there are only $n-r$ independent initial conditions. Since, in general we must have $n$ independent initial conditions, we conclude that $r=0$. Therefore, $A=0$, that is the stiffnesses in states $S^{1}$ and $S^{2}$ are the same. These stiffnesses are: the compound stiffnesses $\left[k\left(x_{p}-x_{p-1}\right)+k\left(x_{p+1}-x_{p}\right)\right]$ and the stiffnesses $k\left(x_{p}-x_{p+1}\right), k\left(x_{p+1}-x_{p}\right)$. These stiffnesses coincide in two states where the trajectories only differ in their signs. This implies that the following relations should hold between the stiffnesses as functions of the differences between the trajectories

$$
\begin{aligned}
& k\left(x_{p}-x_{p-1}\right)+k\left(x_{p+1}-x_{p}\right)=k\left(x_{p-1}-x_{p}\right)+k\left(x_{p}-x_{p+1}\right), \\
& k\left(x_{p}-x_{p-1}\right)=k\left(x_{p-1}-x_{p}\right), \quad k\left(x_{p+1}-x_{p}\right)=k\left(x_{p}-x_{p+1}\right)
\end{aligned}
$$

The last two equations are impossible to satisfy, unless the corresponding springs are in neutral positions. However, as explained earlier this situation cannot last and for that reason is not considered.

Consequently, only when $n=1$ system of Eqns. (12) does not include the last two conditions. This brings us to a single mass chain, Figure 3, considered in the previous section, which was shown to form an ordinary linear oscillator. 
This single mass elementary chain can also form at the middle of a chain by forcing two masses to be stationary and splitting the whole chain into 3 subsystems, Figure 6 b.

The conclusion of this stage is: for an odd number of masses $(n=2 m+1)$ three cases are possible: (1) If the symmetry transformation leads to the phase shift equal to the whole period, the only stable motion corresponds to the central mass being stationary such that the system is split into two independent systems of $m$ masses. Since $m$ can be even, further size reduction requires the analysis of the case of systems with even number of masses, see below. (2) If the symmetry transformation leads to the half period phase shift, the only stable cases are the single mass bilinear chain $(n=1)$, Figure 3, which degenerates to an ordinary linear oscillator or the system is split into three subsystems where the central one being a single mass bilinear chain, Figure $6 \mathrm{~b}$.

\subsection{Even number of masses}

Consider now the case of even number of masses, $n=2 m$, Figure 5. Consider transformation $\gamma_{m}$ that swaps two central masses. Again two cases should be considered.

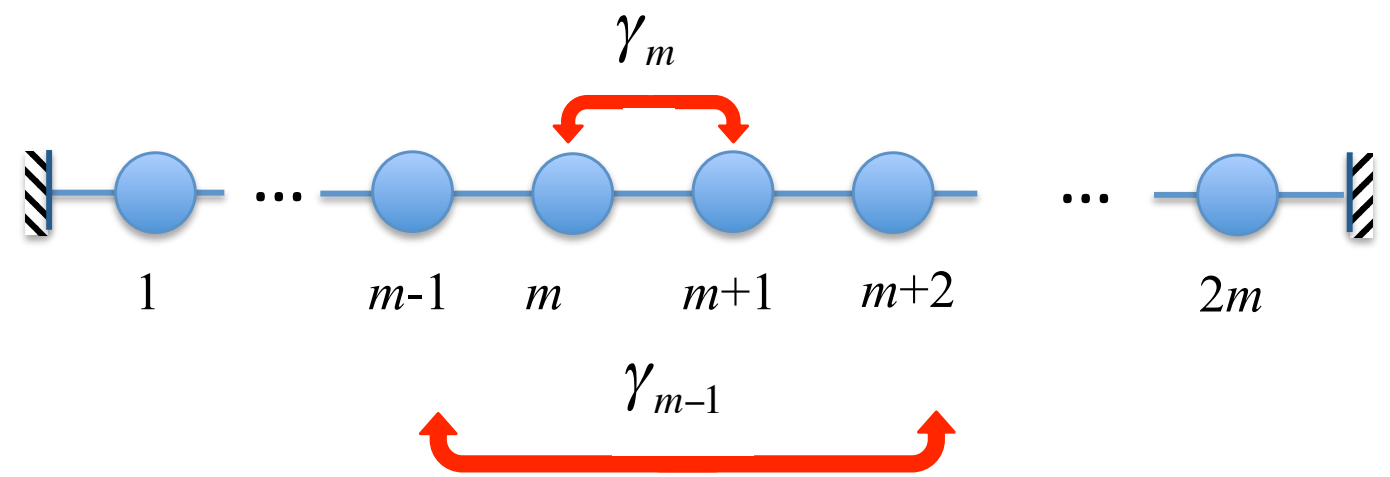

Figure 7. Chain consisting of even number of masses (the curved arrows show particular transformations). 


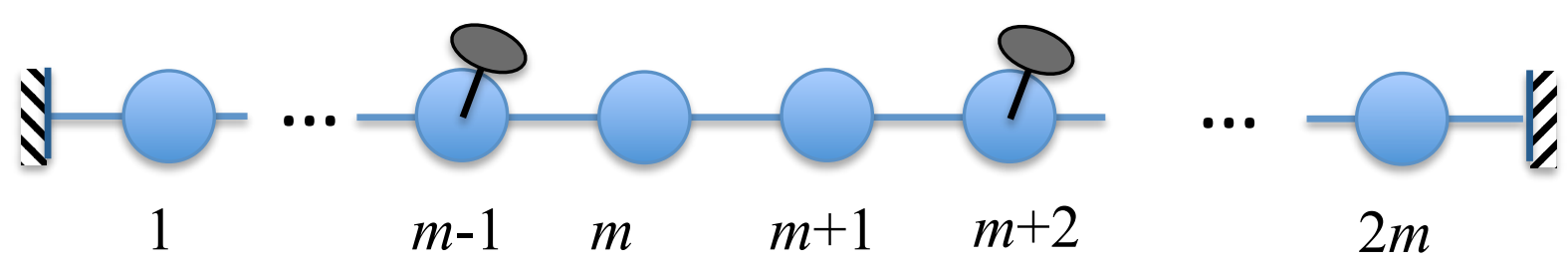

Figure 8. Splitting of a $2 m$ mass chain into three independent chains. The 'nails' in the picture mark the masses that remain stationary in splitting the system into smaller ones.

Case 1: $\gamma_{k} \mathbf{x}(t)=\mathbf{x}(t)$, for some $k=1, \ldots, m$.

Then $x_{m+1}(t)=x_{m}(t)$, i.e. the central spring is neither in tension nor in compression. This state is obviously not sustainable; a small disturbance will violate this equality.

\section{Case 2:}

Let $m>1$. Suppose $\gamma_{k} \mathbf{x}(t)=\mathbf{x}(t+T / 2), \forall k=1, \ldots, m$. Then the successive application of $\gamma_{m}$ and $\gamma_{m-1}$, Figure 7, brings us to Case 1: $x_{m+1}(t)=x_{m}(t)$, which is unsustainable. The only other possibility to consider is $m=1$. In this case $\gamma_{m-1}$ does not exist, so it is not possible to reduce this case to Case 1 . This brings us to another elementary chain - a two mass bilinear oscillator, Figure 1. Naturally this two mass chain can be formed in the middle of the chain by forcing two masses to be stationary, Figure 8 . The whole chain is then split into three subsystems: the central chain that involves 4 masses and two chains of $m-2$ masses.

\subsection{Decomposition rule}

We now note that the eigenfrequencies of the single mass bilinear chain, Figure 3 and the coupled oscillator, Figure 1 are different. Therefore, if a chain were to be split into a combination of single and coupled oscillators the dividing masses could not be kept stationary. This circumstance imposes restriction on the length of the chains that support resonances. The length must be either odd, $n=2 m+1$, in which case it can be split into $m+1$ single mass bilinear chains or $n=3 k+2$, then the chain is split into $k+1$ two mass bilinear chains. Chains of all other lengths do not support resonances. 
An interesting special case is when the chain length satisfies both conditions, $n=2 m+1=3 k+2$. In this case resonances are possible on both frequencies. For this to happen $k+1$ should obviously be even, therefore the double resonance frequency case corresponds to the chain lengths $n=6 l-1$, $l=1, \ldots$. The possible decompositions are summarised as follows

$$
n=\left\{\begin{array}{l}
2 m_{1}+1, m_{1}=0, \ldots-\text { decomposition into single mass bilinear chains } \\
3 m_{2}+2, m_{2}=0, \ldots \text { - decomposition into two mass bilinear chains } \\
6 l-1, l=1, \ldots-\text { decomposition into either single or two mass bilinear chains }
\end{array}\right.
$$

Here $m_{1}+1$ is the number of single mass bilinear chains in the decomposition with $m_{1}$ stationary masses, $m_{2}+1$ is the number of two mass bilinear chains in the decomposition with $m_{2}$ stationary masses.

If both decompositions are possible, which one actually happens is determined by the driving frequency: if the driving frequency is equal to the eigenfrequency of the single mass bilinear chain then the chain will be decomposed into $m_{1}+1$ single mass bilinear chains with $m_{1}$ stationary masses. If the driving frequency is equal to the eigenfrequency of the two mass bilinear chain (or its multiple) then the chain will be decomposed into $m_{2}+1$ two mass bilinear chains with $m_{2}$ stationary masses.

\subsection{Resonance frequencies}

The above analysis shows that the chain of bilinear oscillators has either one or two or no eigenfrequencies depending upon the number of oscillators in the chain. This corresponds to the possibility of the chain oscillations to decompose into the oscillations of the first basic oscillators (a mass on two bilinear springs) or the second basic (coupled bilinear) oscillators. The first basic oscillator, Figure 3 behaves as a conventional linear oscillator. Therefore, as long as the chain oscillations get decomposed into oscillations of the first basic oscillator, the eigenfrequency becomes (the only) resonance frequency. The second basic oscillator, Figure 1, has only one eigenfrequency (8), which coincides with the frequency of a bilinear oscillator with the corresponding stiffnesses of the bilinear spring. Strictly speaking, since the oscillators are nonlinear the eigenfrequencies do not have to correspond to resonance frequencies. It was shown through numerical experiments [29, 42] that these frequencies correspond to resonances. 
Furthermore, resonances are detected on frequencies multiple and fractional to the eigenfrequency of the bilinear oscillator.

\section{Numerical examples}

In this section we consider some examples of resonances of chains of bilinear oscillators. We analysed and simulated the structure of the resonances in the chains of 2 to 12 masses. We assume, following [42], the nonlinearity factor $K=20$ and $\omega_{+}=1$.

Table 2 lists the resonant frequencies and modes obtained for the chains with $n=1$ to 12 masses. It shows the resonance frequencies (when exist) of the first 12 chains of $n$ bilinear oscillators and the components of the common eigenvectors of the states whose alternation enables the resonances. The relations between the chain length and the decompositions correspond to Eqn. (13). It is seen that all resonance frequencies coincide with the resonance frequency of one of the basic chains either the single mass bilinear chain or the two mass bilinear chain. Furthermore, the resonance modes have stationary points (masses), which do not move during the oscillations. These stationary points break the whole system into a number of either single or two mass bilinear chains, but not into any combination thereof. The chains of 4, 6, 10 and 12 masses cannot be split into the basic chains and consequently they have no resonances. On the other hand, the five mass and eleven mass chains can be decomposed into either single mass or two mass bilinear chains and thus possess two resonance modes. 
Table 2. Common eigenvectors and resonant frequencies in the chains of $n$ bilinear oscillators with the factor of non-linearity $K=20$.

\begin{tabular}{|c|c|c|c|c|c|c|c|c|c|c|c|c|}
\hline \multirow{2}{*}{$n$} & \multirow{2}{*}{ Resonant frequency } & \multicolumn{11}{|c|}{ Components of the common eigenvector } \\
\hline & & 1 & 2 & 3 & 4 & 5 & 6 & 7 & 8 & 9 & 10 & 11 \\
\hline 1 & 4.5826 & $(-1)$ & & & & & & & & & & \\
\hline 2 & 5.4146 & $(-1$ & 1) & & & & & & & & & \\
\hline 3 & 4.5826 & $(-1$ & 0 , & 1) & & & & & & & & \\
\hline 4 & No resonance & & & & & & & & & & & \\
\hline - & 4.5826 & $(-1$ & 0 , & 1 , & 0 & $-1)$ & & & & & & \\
\hline & 5.4146 & $(-1$ & 1 , & 0 , & -1 , & 1) & & & & & & \\
\hline 6 & No resonance & & & & & & & & & & & \\
\hline 7 & 4.5826 & $(-1$ & 0 & 1 , & 0 & -1 , & 0 , & 1) & & & & \\
\hline 8 & 5.4146 & $(-1$ & 1 , & 0 , & -1 , & 1, & 0 , & -1 , & 1) & & & \\
\hline 9 & 4.5826 & $(-1$ & 0 & 1 , & 0 & -1 , & 0 , & 1, & 0 , & $-1)$ & & \\
\hline 10 & No resonance & & & & & & & & & & & \\
\hline 11 & 4.5826 & $(-1$ & 0 & 1, & 0 & -1 , & 0 , & 1 , & 0 & -1 , & 0 & 1) \\
\hline & 5.4146 & $(-1$ & 1 , & 0 , & -1 , & 1, & 0 , & -1 , & 1, & 0 & -1 , & 1) \\
\hline 12 & No resonance & & & & & & & & & & & \\
\hline
\end{tabular}

Table 3 shows the resonance eigenmodes together with their respective decompositions that demonstrate the way the system is broken by stationary points into the basic oscillators. The open circles indicate the initial (neutral) state of the masses; the filled in circles indicate current positions of the masses in the eigenmodes. The position of the filled in circle to the right of the open circle corresponds to the component +1 of the eigenvector in Table 2 , while the position of the filled in circle to the left of the open circle corresponds to the component -1 of the eigenvector. The position of the filled in circle coinciding with the open one indicates a stationary point; this corresponds to component 0 of the eigenvector in Table 2. 
Table 3. Composition of the resonance modes in the chains of bilinear oscillators (Symbols x2, x3, $\mathrm{x} 4$ signify the repetition of the whole sub-chain in the right column 2, 3 or 4 times respectively).

Resonance mode

As can be seen from Table 3, each resonance mode corresponds to the chain decomposition into a number of basic chains of the same type, as per Eqn. (13). The chain then inherits the resonance frequency of the basic chains it decomposes into. For example, the resonance mode of a three-mass oscillator is obtained by decomposing it into two single mass bilinear chains, with the middle mass being stationary. At the same time, the four mass chain does not have a resonance, since it does not allow decomposition into any combination of identical basic chains.

Based on the above decomposition rules, the number of masses in the chains corresponding to each of the basic oscillators can be determined as follows. Suppose the chain is decomposed into $m_{1}+1$ single mass bilinear chains (the first basic oscillator) separated by $m_{1}$ stationary points. Therefore the total number of masses is $n=2 m_{1}+1$. Thus all chains with odd numbers of masses are 
decomposable into a series of single mass bilinear chains and hence inherit their resonance frequency of 4.5826 .

If the chain is decomposed into $m_{2}+1$ two mass bilinear chains separated by $m_{2}$ stationary points, the total number of masses is $n=3 m_{2}+2$. Subsequently all chains with these numbers of masses are decomposable into a series of two mass bilinear chains and hence inherit their resonance frequency of 5.4146. These are the cases of $n=5,8,11$ shown in Tables 2 and 3.

When the number of masses $n$ satisfies the above relationships simultaneously, both decompositions are possible, and both frequencies 4.5826 and 5.4146 are resonance frequencies. Such are the cases of $n=5,11$, see Tables 2 and 3 .

In both cases the number of stationary points is

$$
m_{\text {Stationary points }}=m_{i}, \quad i=1,2
$$

where $m_{1}+1$ is a number of the single mass bilinear chains, $m_{2}+1$ is a number of the two mass bilinear chains, and $m_{\text {Stationary points }}$ is a number of the points remained stationary in the chain.

Since the double mass bilinear chain behaves as a single bilinear oscillator with a certain effective resonance frequency, it should exhibit super- and sub-harmonic resonances (e.g., [5]). We now examine the presence of super- and sub-harmonic resonances in $n$-mass chains of bilinear oscillators. In order to illustrate the resonance behaviour of the chains of bilinear oscillators, we consider 4 chains with 3, 4, 5, and 8 masses respectively, which covers all possible cases shown in Tables 2 and 3. In all examples we use following parameters: $M=1, K=20$ and $\omega_{+}=1$. This leads to two possible resonance frequencies $\Omega_{r}=4.5826$ and $\Omega_{r}=5.4146$, Table 2 .

Figure 9 shows the oscillations and frequency spectrum of a chain of three bilinear oscillators harmonically excited with the frequencies $\Omega$ multiple to and half of the fundamental resonant frequencies, $\Omega_{r}=4.5826$ corresponding to a single mass bilinear chain. In order to produce resonance, we excite the chains in accordance with the obtained resonance mode, Table 2. For instance, in the case of three mass chains, we apply harmonic forces with the same frequency in the 
direction specified by the eigenvector $(-1,0,1)$. It is seen that out of four cases presented in Figures $9 \mathrm{a}-\mathrm{d}$, the system only responds with resonance when excited at the fundamental frequency, Figure 9a. The multiple frequencies of $2 \Omega_{R}, 3 \Omega_{R}$, and $0.5 \Omega_{R}$ merely lead to periodic solutions. Furthermore, the spectra corresponding to $0.5 \Omega_{R}$, and $2 \Omega_{R}$ excitations only show the fundamental and excitation frequencies, Figure $9 \mathrm{e}$ and $\mathrm{f}$. This kind of behaviour is typical for linear oscillators. This is not surprising since the chain of this length has resonance decomposition into a pair of identical single mass bilinear chains each behaving as a linear oscillator, Table 3.

Figure 10 presents the next case, a chain with four masses. Since this chain is not supposed to exhibit resonances, we excite this system using both fundamental frequencies obtained in Table 2 with the same eigenvector of $(0,1,-1,0)$. It can be observed that the chain response does not exhibit resonance, Figure $10 \mathrm{a}, \mathrm{b}$. The frequency spectrum constructed using these amplitudes is a spectrum of a chaotic motion, Figure $10 \mathrm{c}, \mathrm{d}$. Note that direct numerical simulations shows that this system does not exhibits any resonances.

Next we consider a chain of five masses, which supports two types of decomposition, either into 2 two mass bilinear chains with a stationary point in the middle or 3 single mass bilinear chains with 2 stationary points in between them, Table 3. Figures 11 and 12 show the response of the system to the excitation forces with following frequencies: $\Omega_{R}, 2 \Omega_{R}, 3 \Omega_{R}$, and $0.5 \Omega_{R}$, where $\Omega_{R}$ is the main resonant frequency of the respective mode. The first mode that corresponds to the two mass bilinear chain decomposition is specified by eigenvector $(-1,1,0,-1,1)$, while the second mode of the single mass bilinear chain decomposition corresponds to eigenvector $(-1,0,1,0,-1)$, Table 2 . 


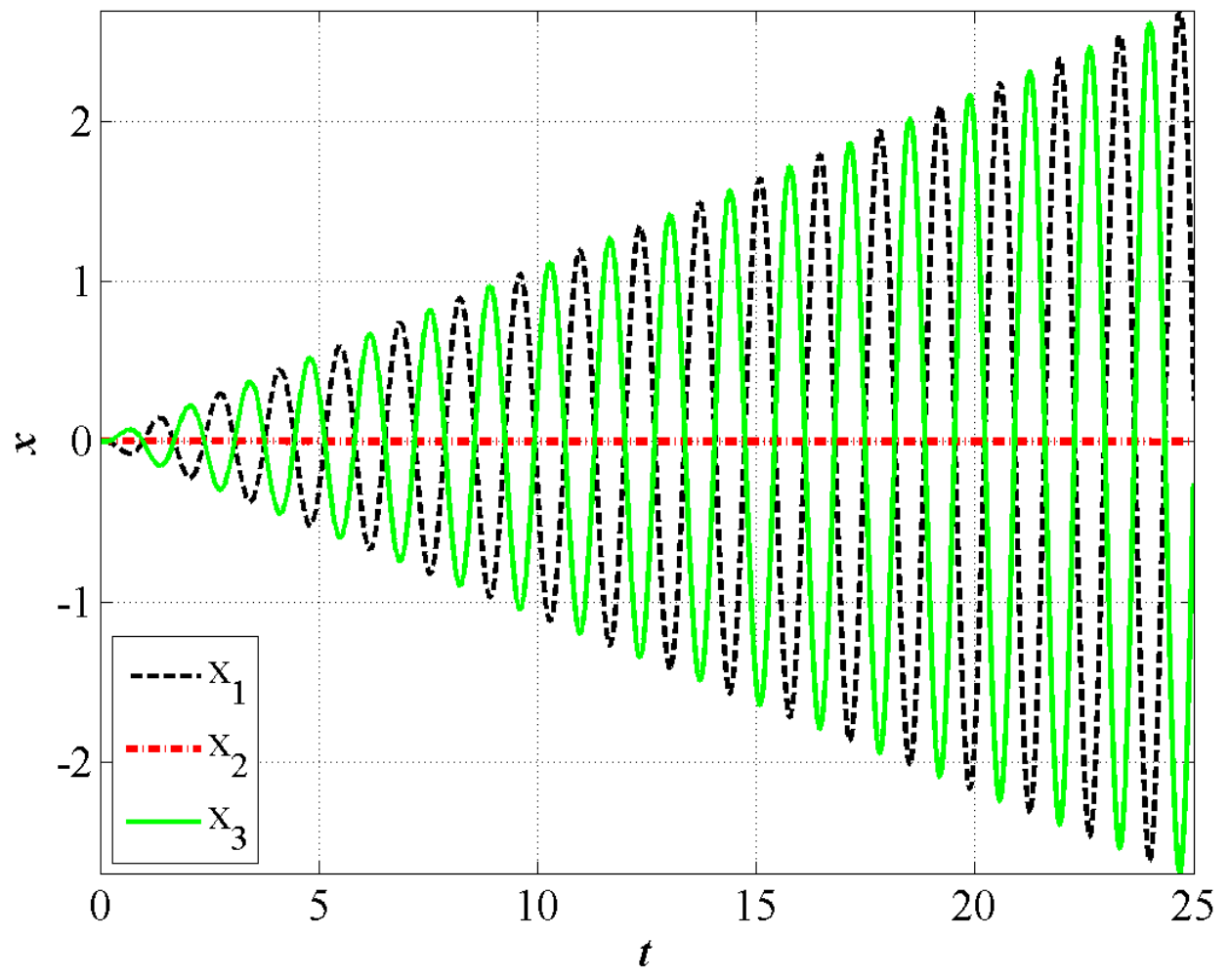

a) Motion for $\Omega=\Omega_{\mathrm{R}}$ 


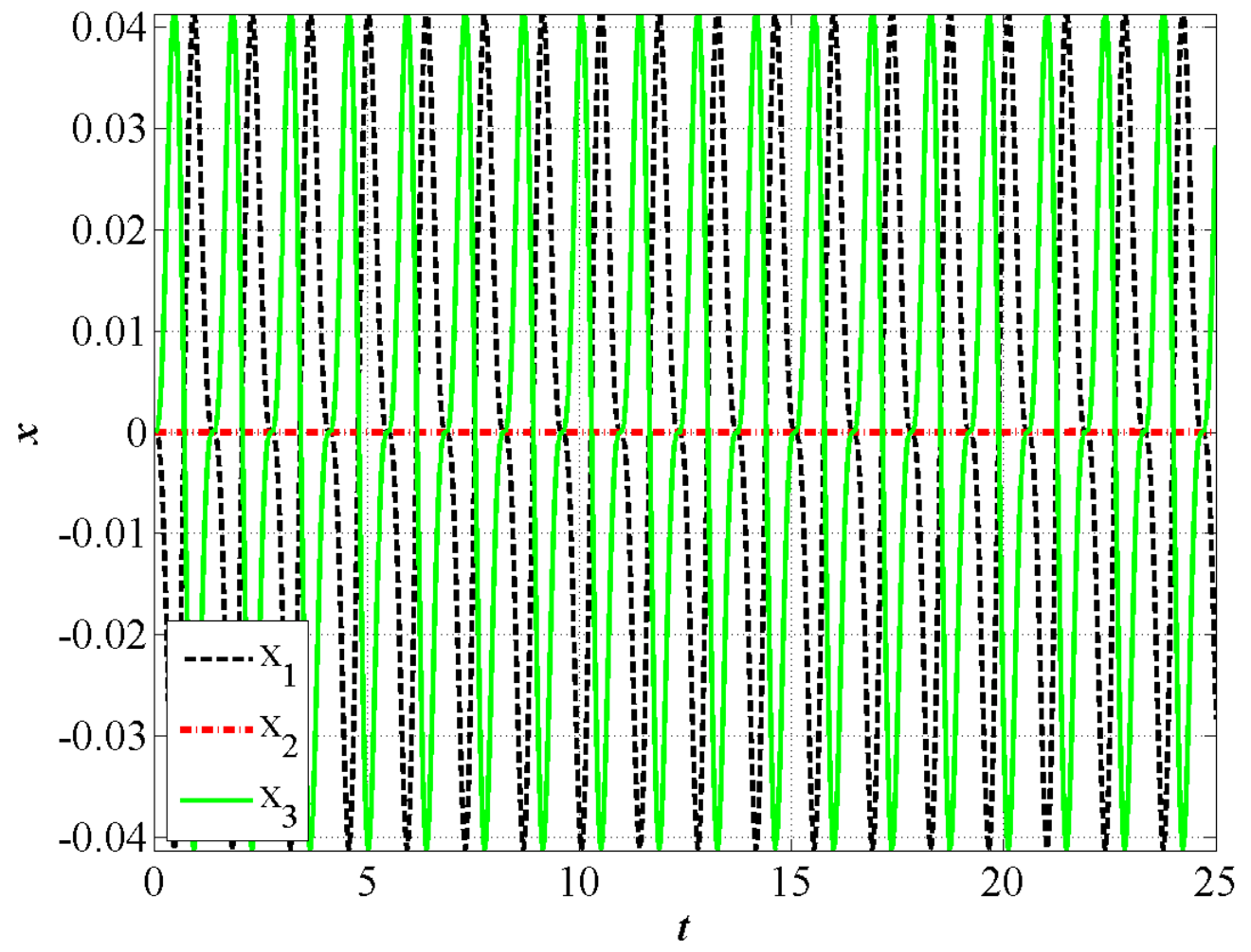

b) Motion for $\Omega=2 \Omega_{\mathrm{R}}$ 


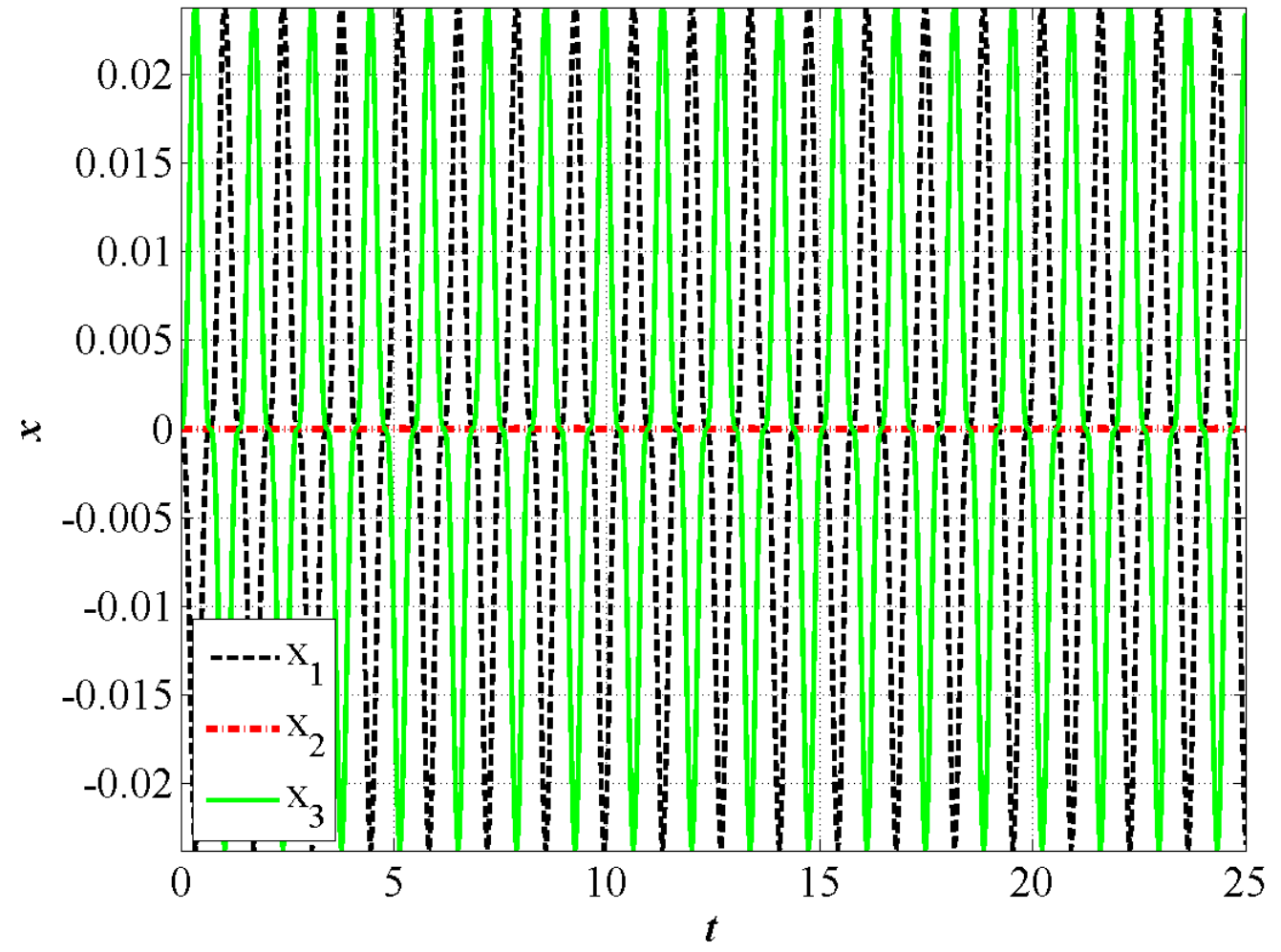

c) Motion for $\Omega=3 \Omega_{\mathrm{R}}$ 


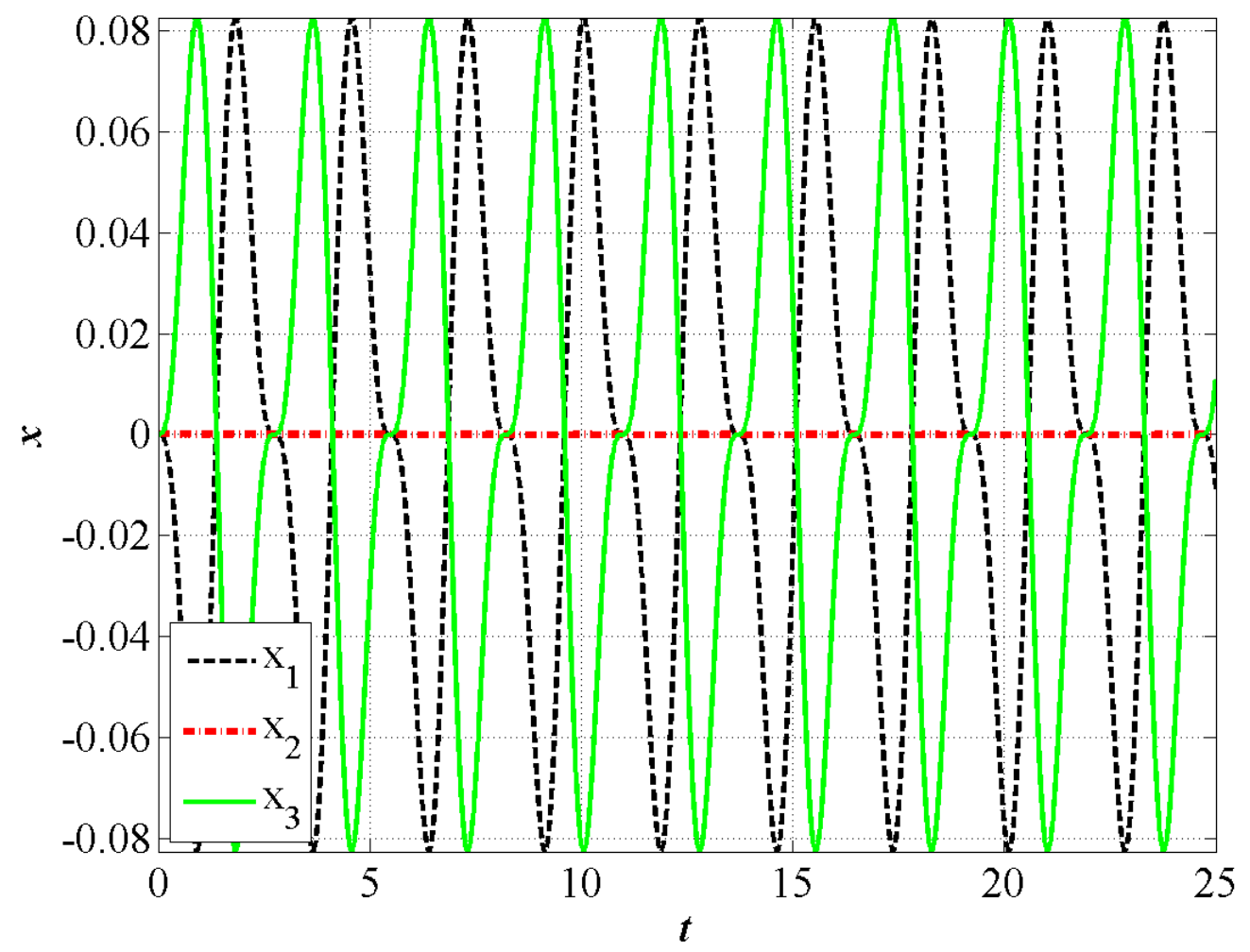

d) Motion for $\Omega=0.5 \Omega_{R}$

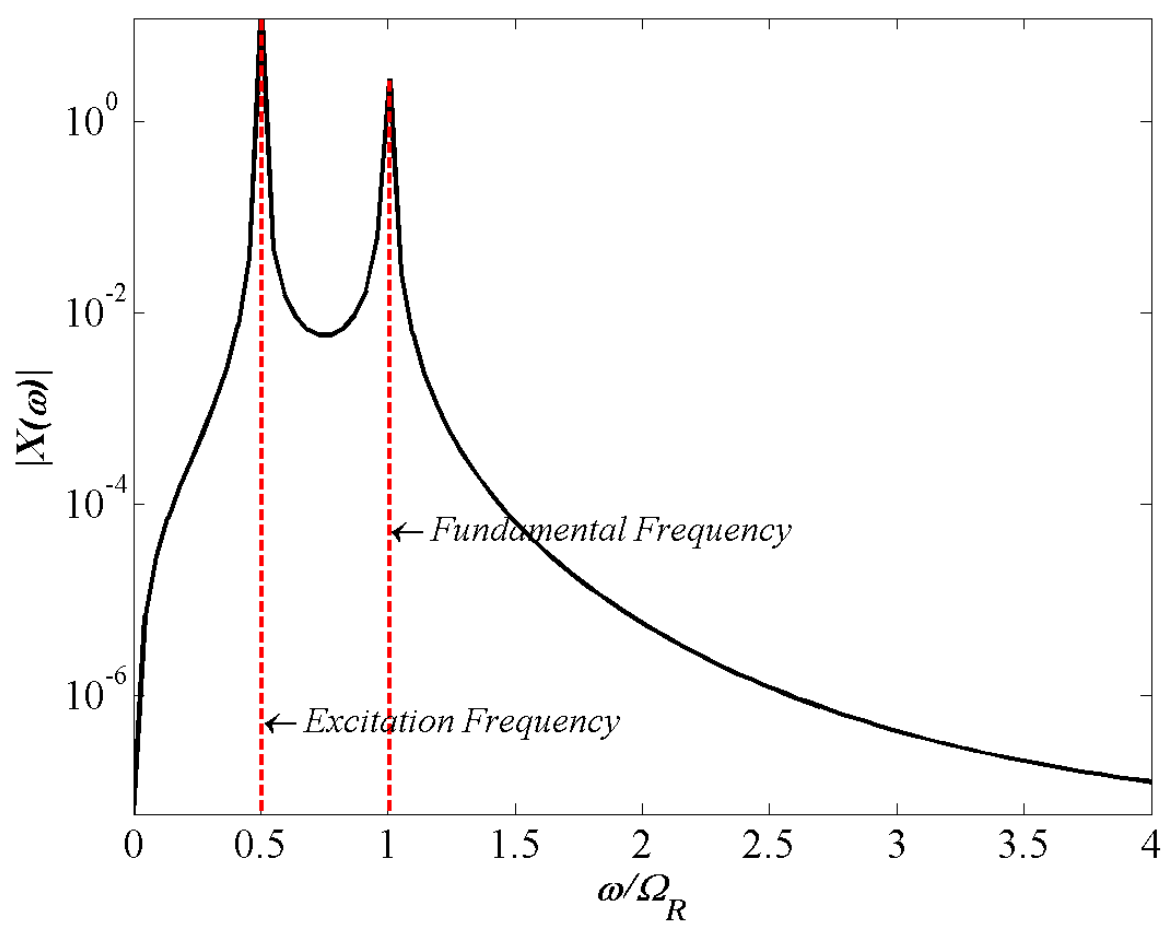

e) Spectrum for $\Omega=0.5 \Omega_{R}$ 


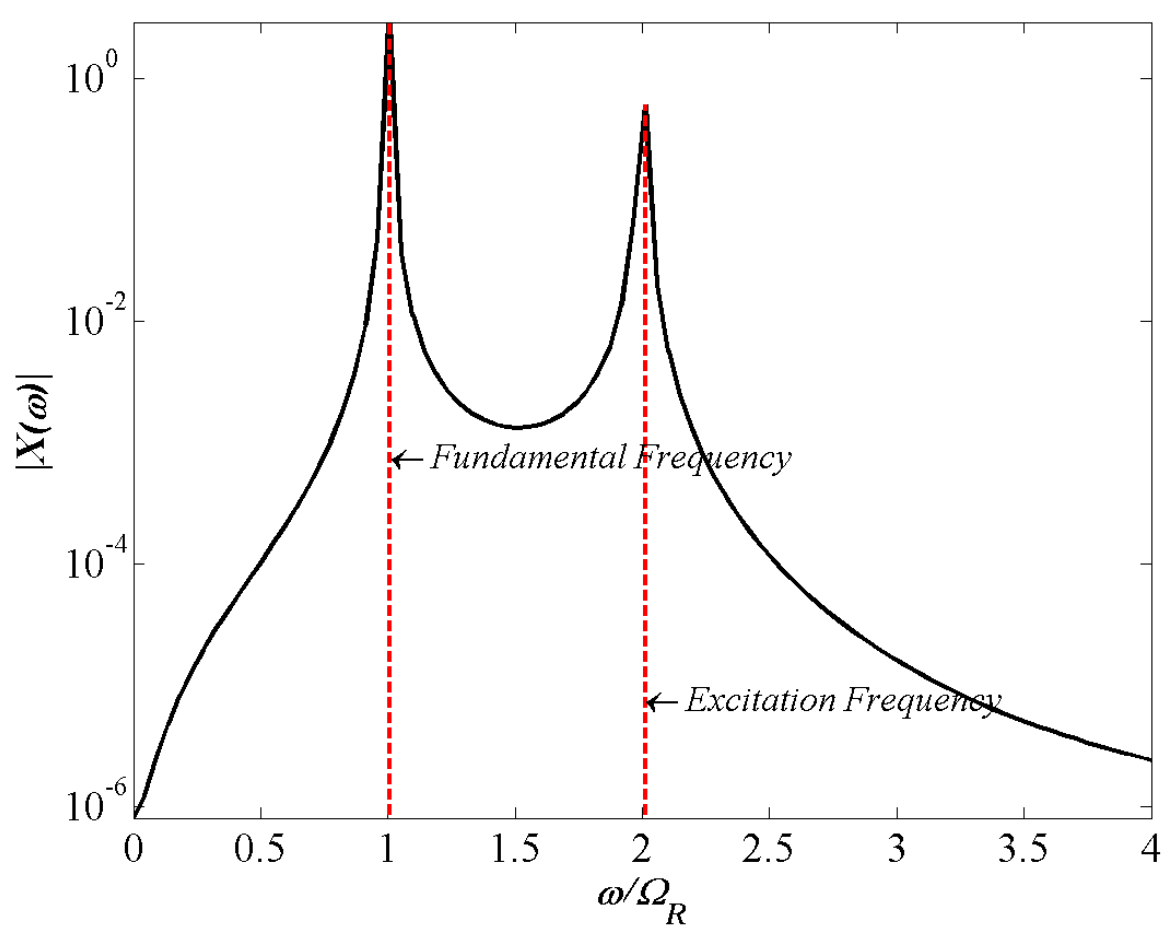

f) Spectrum for $\Omega=2 \Omega_{R}$

Figure 9. A three mass bilinear chain, which permits resonance decomposition into two single mass bilinear chains. The chain is excited by harmonic forces with the same frequency $\Omega$ applied in the directions specified by the eigenvector $(-1,0,1) ; \Omega_{R}=4.5826, K=20, \omega_{+}=1$. 


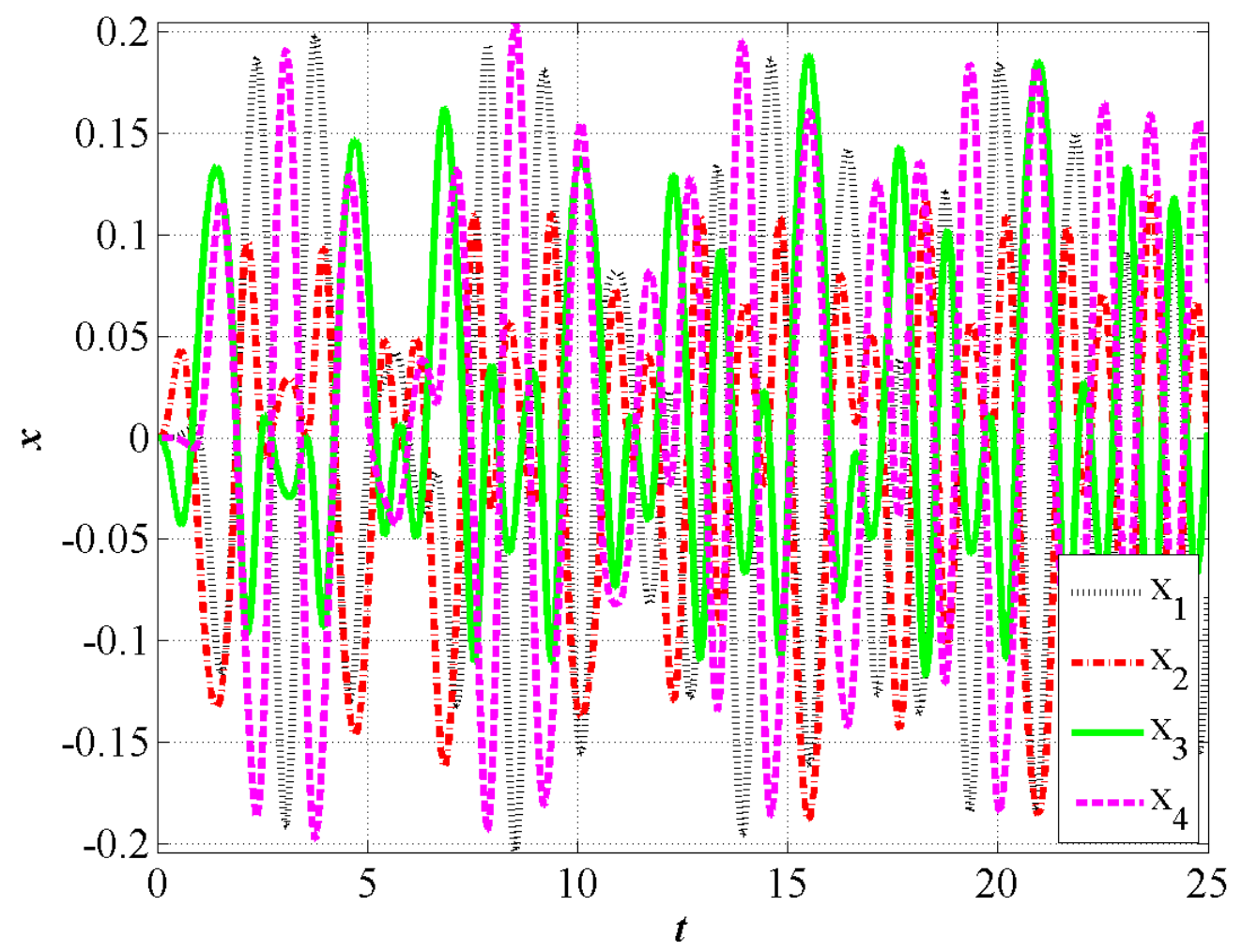

a) Motion for $\Omega=\Omega_{R}=4.5826$ 


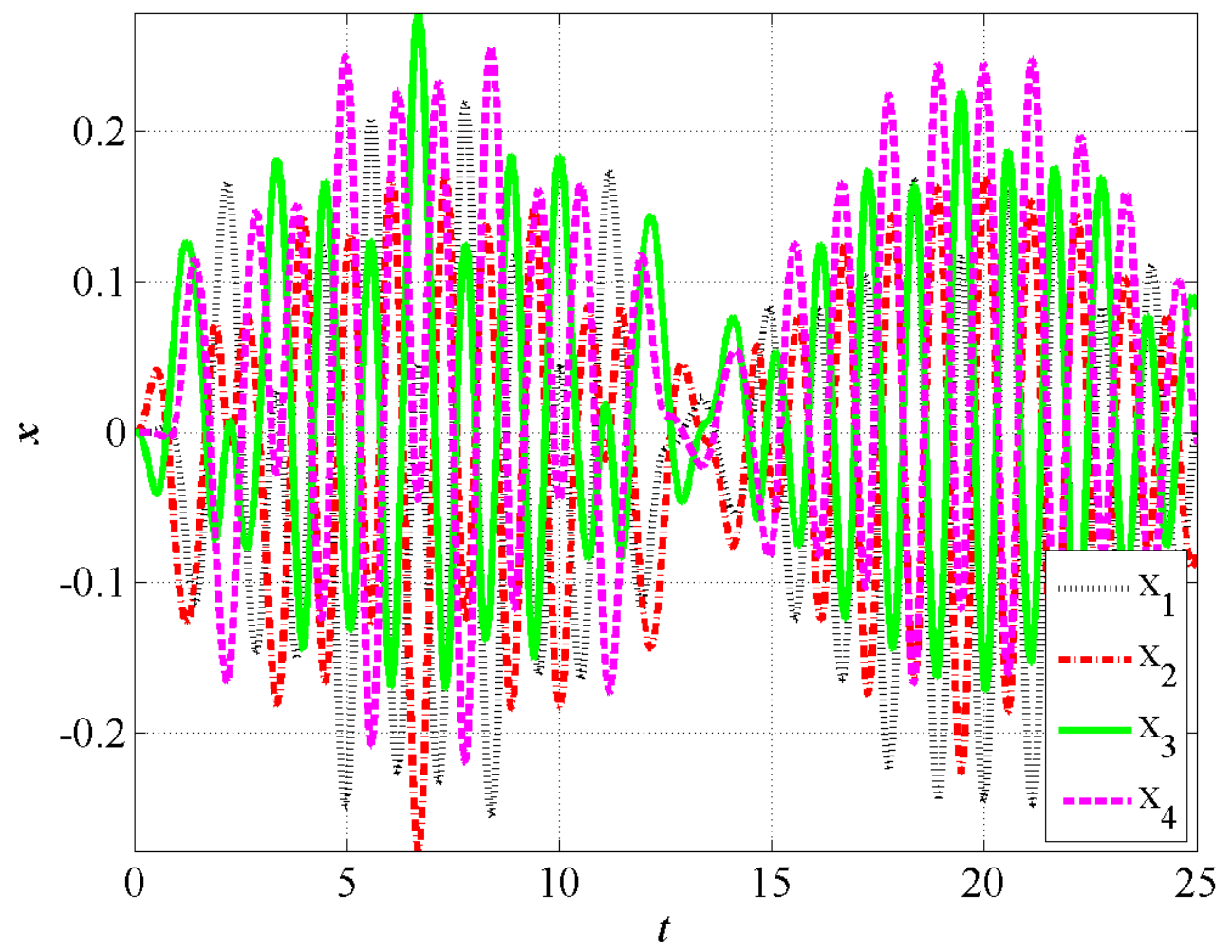

b) Motion for $\Omega=\Omega_{R}=5.4146$

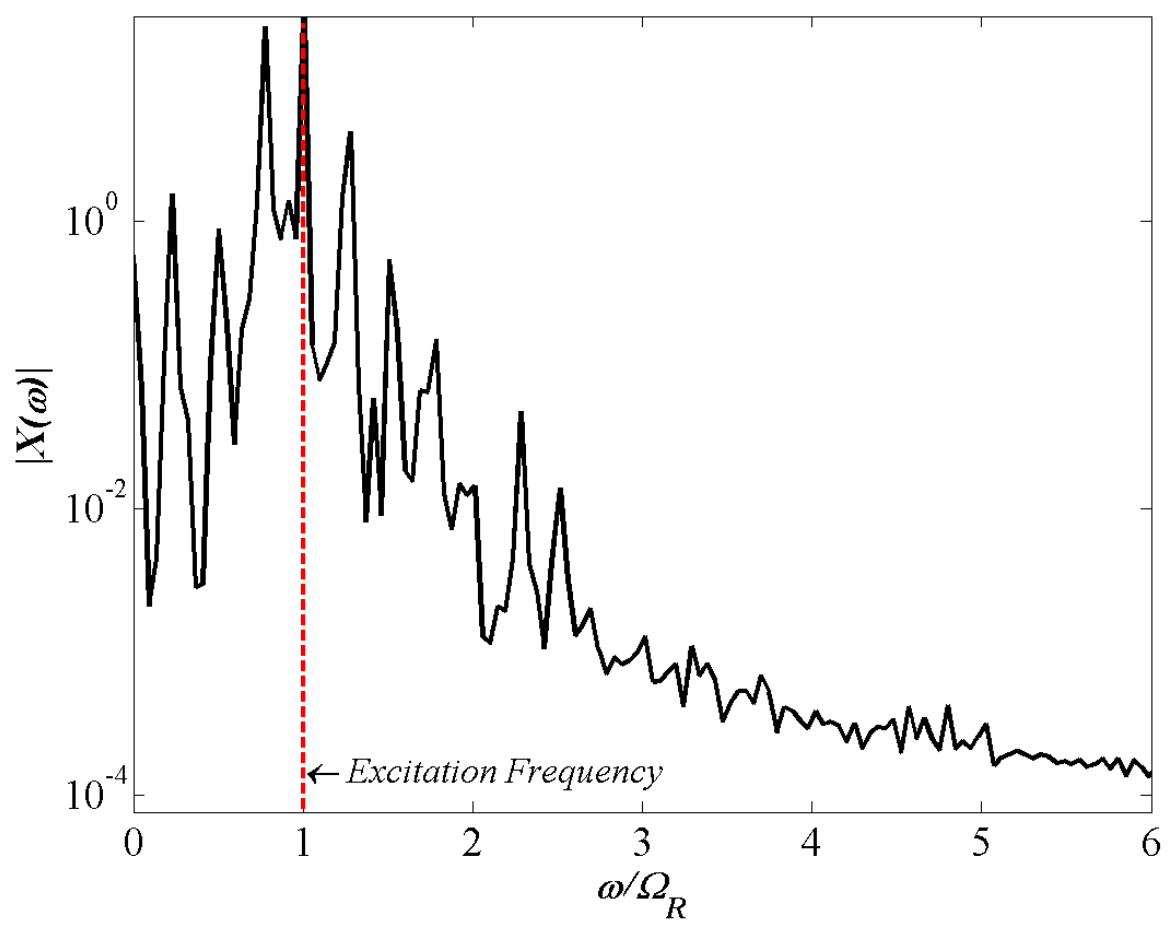

c) Spectrum for $\Omega=0.5 \Omega_{R}$ 


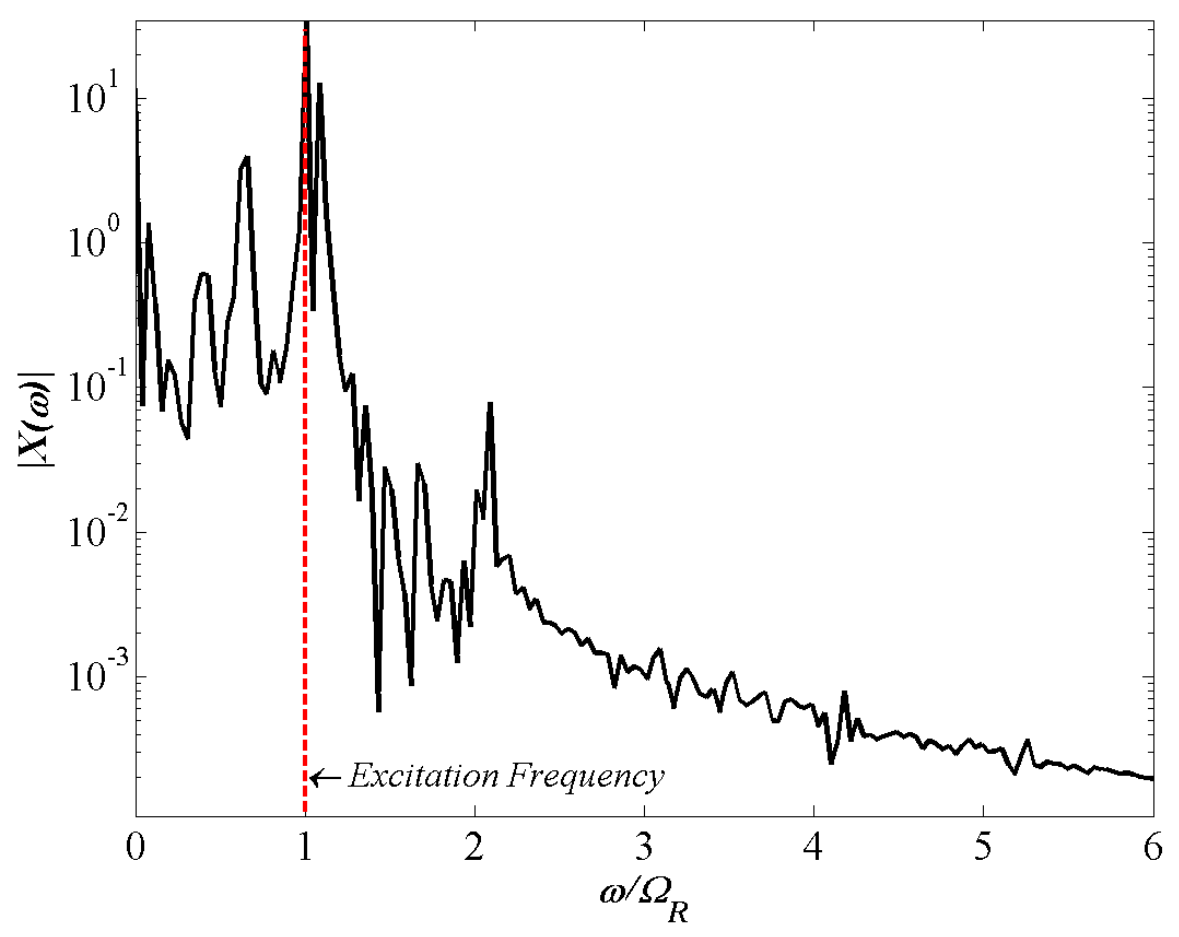

d) Spectrum for $\Omega=2 \Omega_{R}$

Figure 10. A four mass bilinear chain, which does not permit resonance decomposition. The chain is excited by harmonic forces with the same frequency $\Omega$ applied in the directions specified by the eigenvector $(0,-1,1,0), K=20, \omega_{+}=1$. 


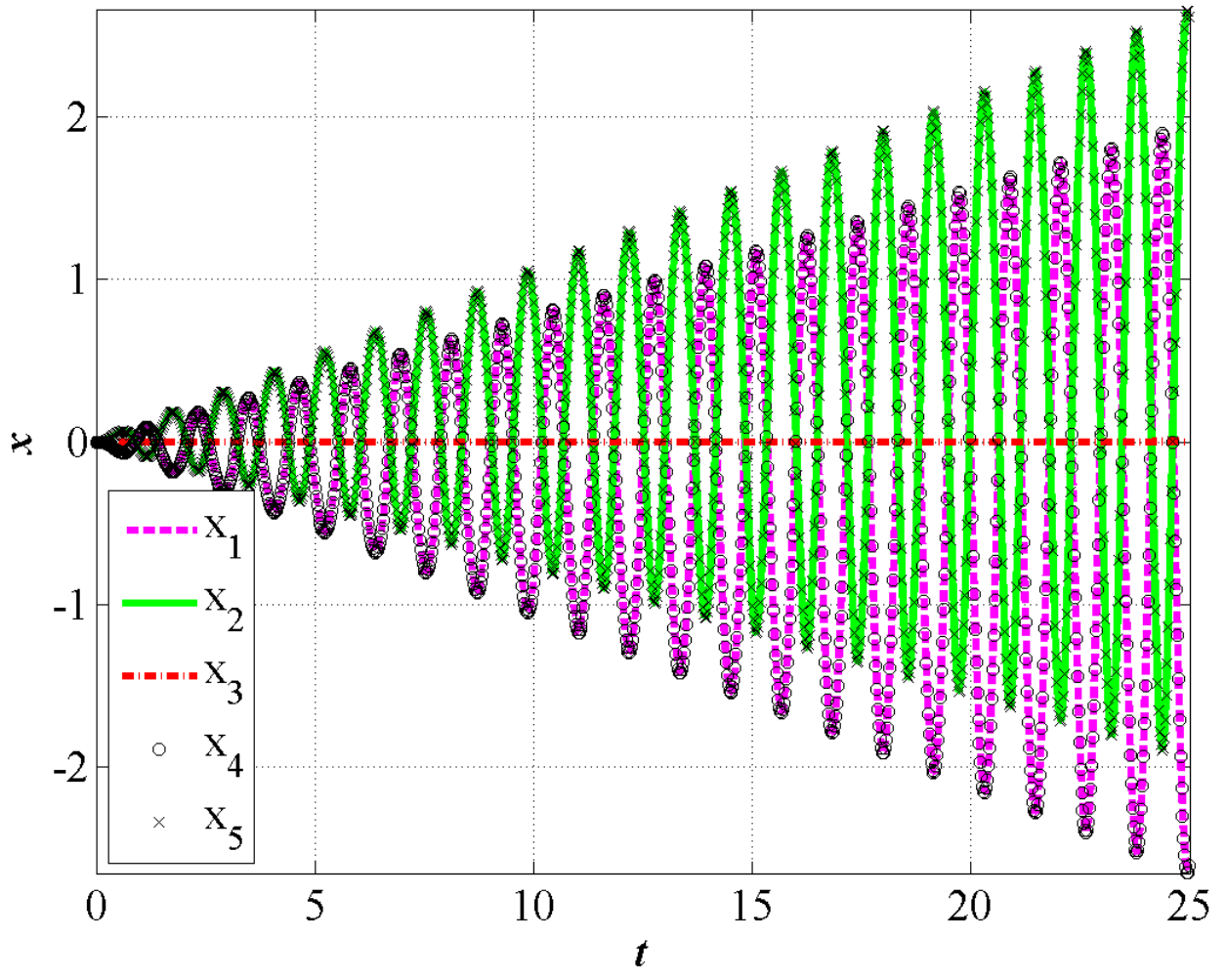

a) Motion for $\Omega=\Omega_{R}$ 


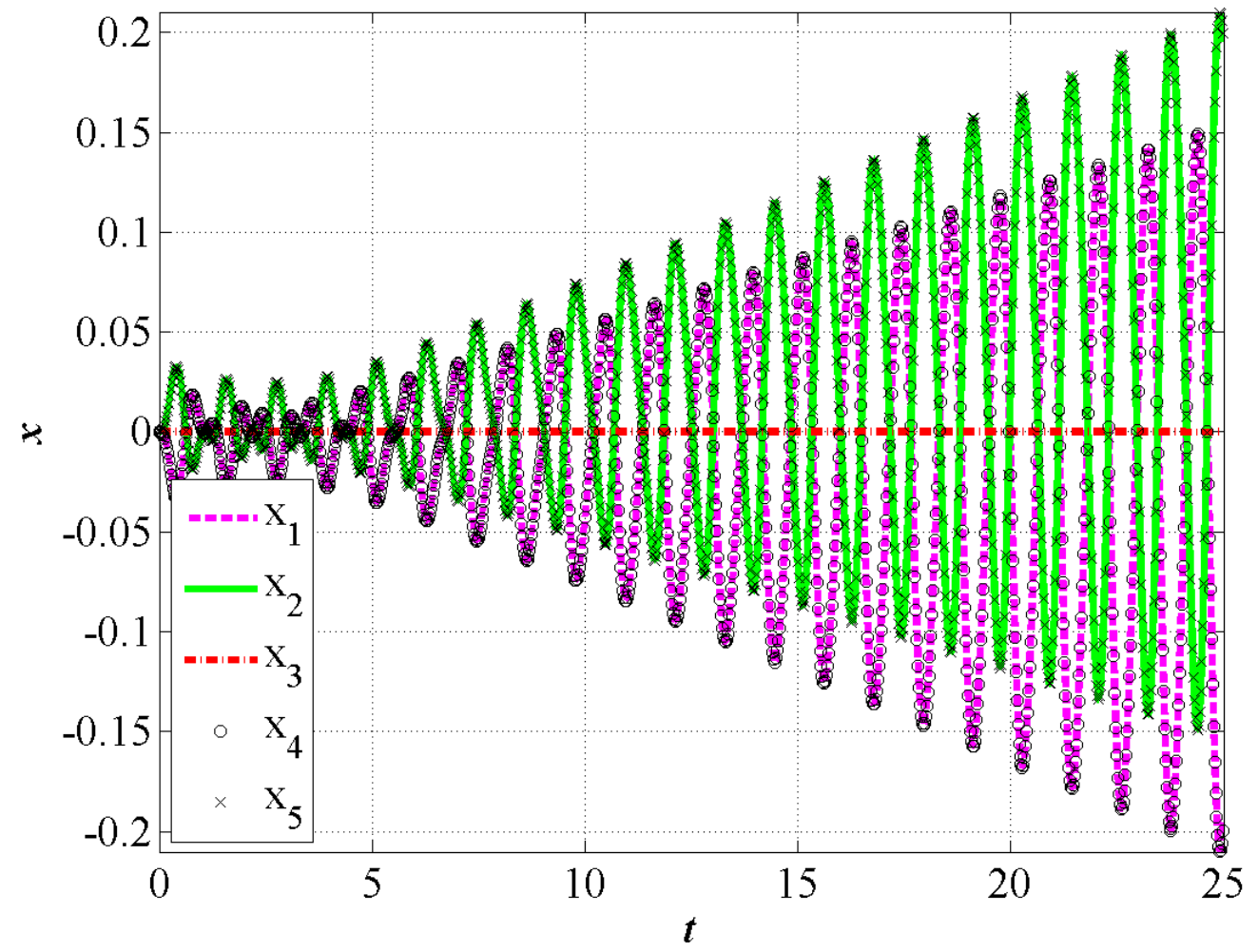

b) Motion for $\Omega=2 \Omega_{\mathrm{R}}$ 


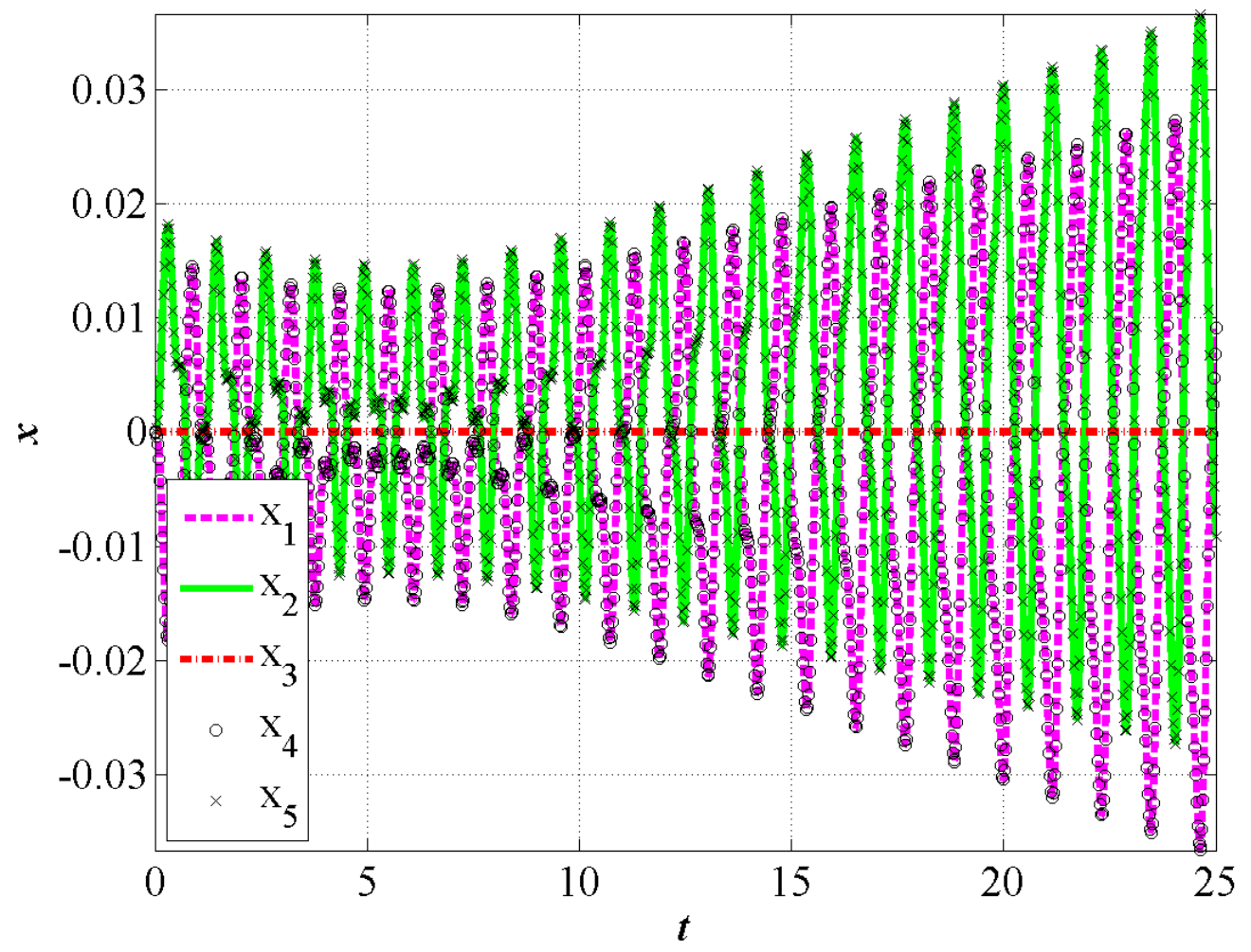

c) Motion for $\Omega=3 \Omega_{\mathrm{R}}$ 


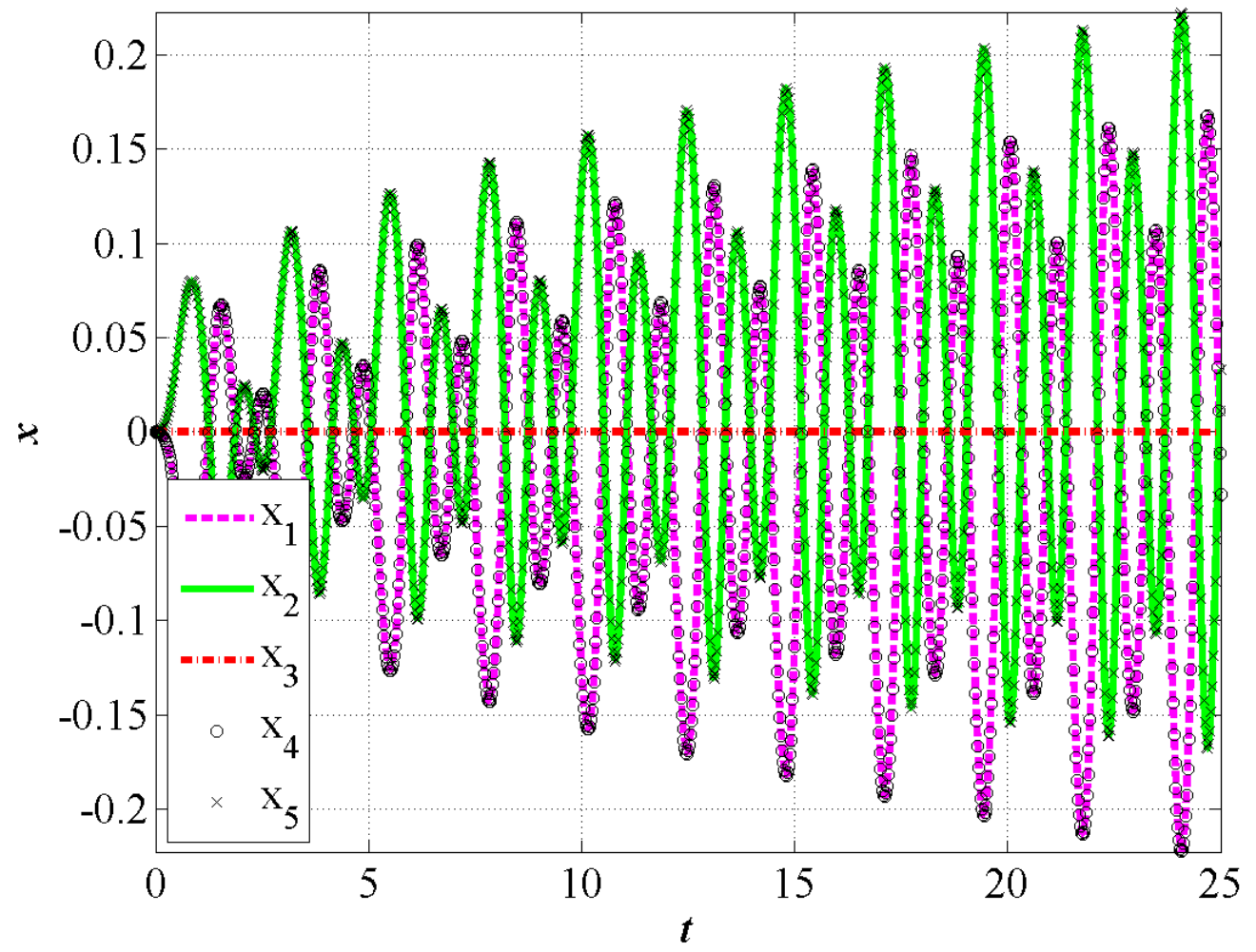

d) Motion for $\Omega=0.5 \Omega_{R}$

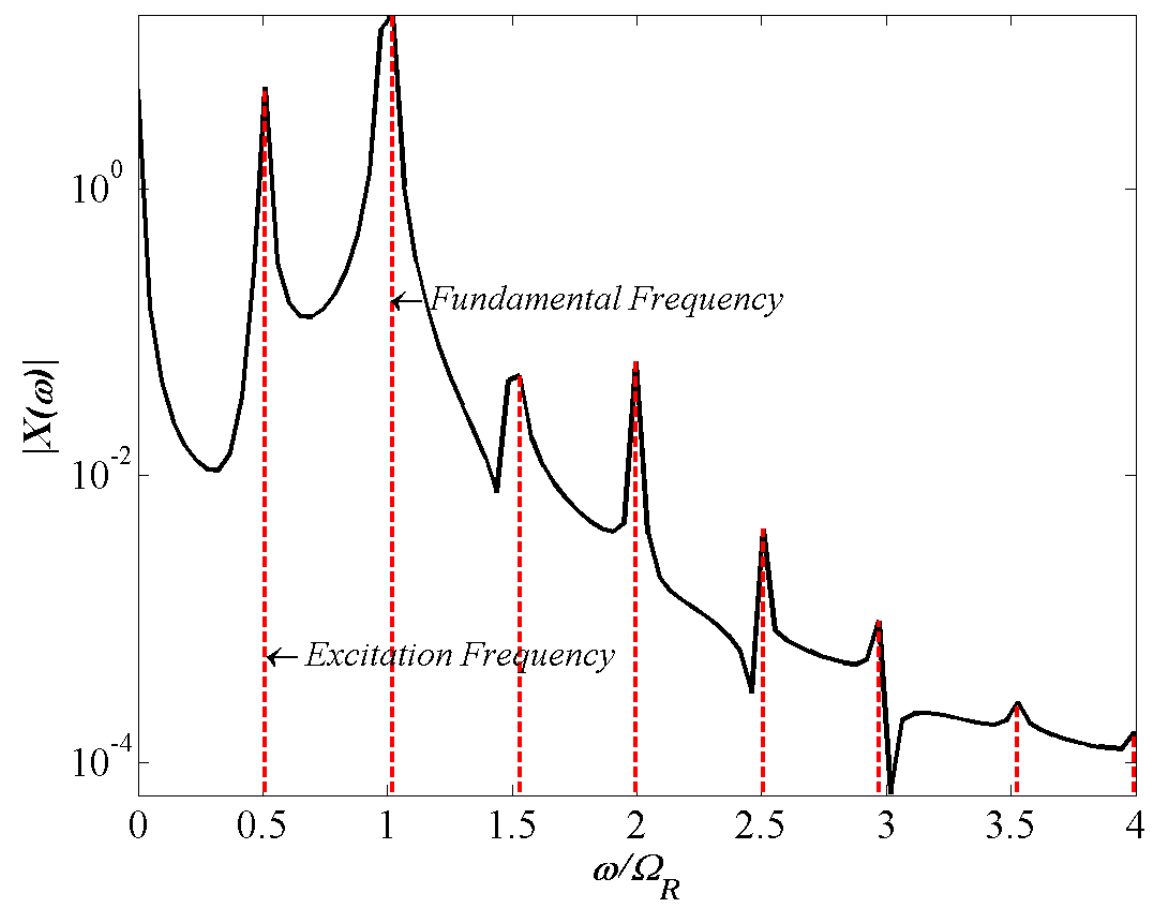

e) Spectrum for $\Omega=0.5 \Omega_{R}$ 


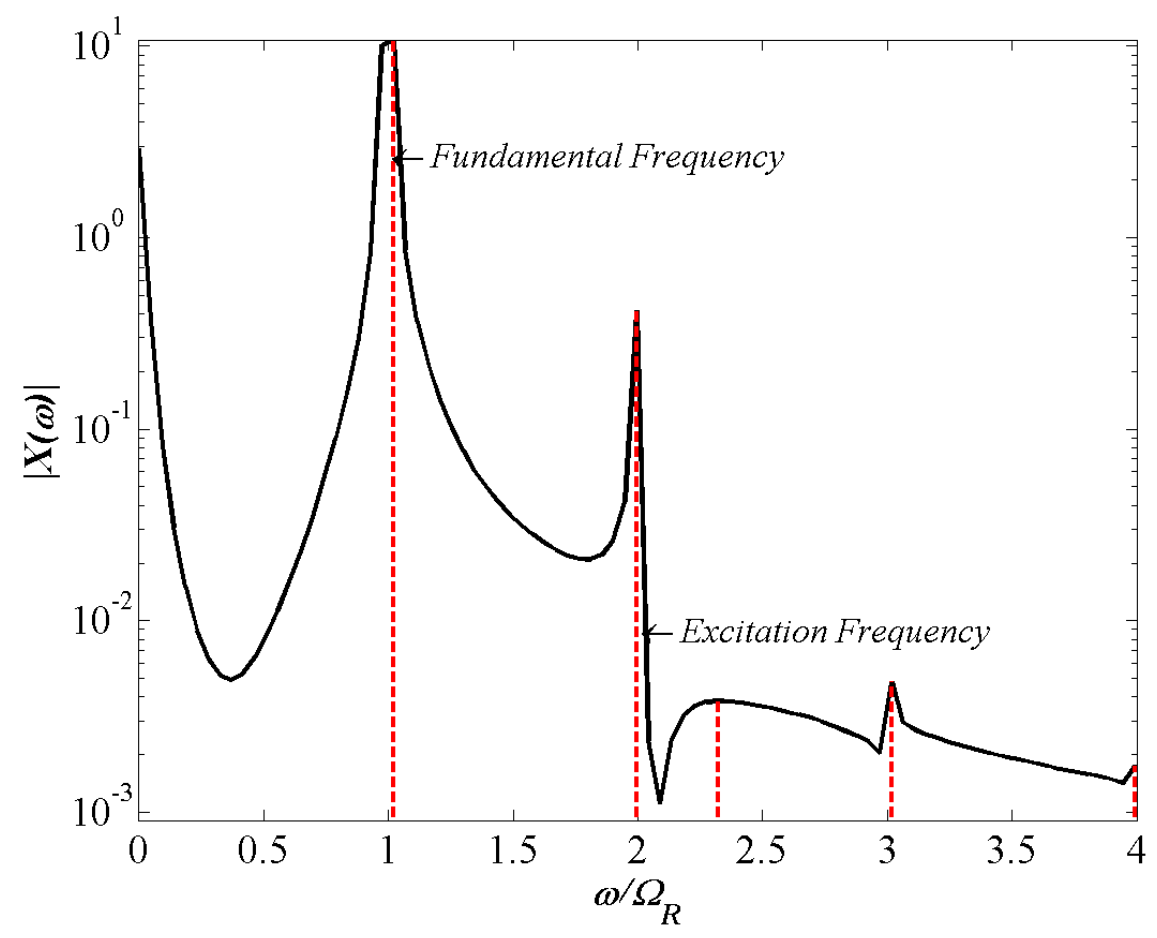

f) Spectrum for $\Omega=2 \Omega_{R}$

Figure 11. A five mass bilinear chain, which permits resonance decomposition into a couple of two mass bilinear chains. The chain is excited by harmonic forces with the same frequency $\Omega$ applied in the directions specified by the eigenvector $(-1,1,0,-1,1), \Omega_{R}=5.4146, K=20, \omega_{+}=1$. 


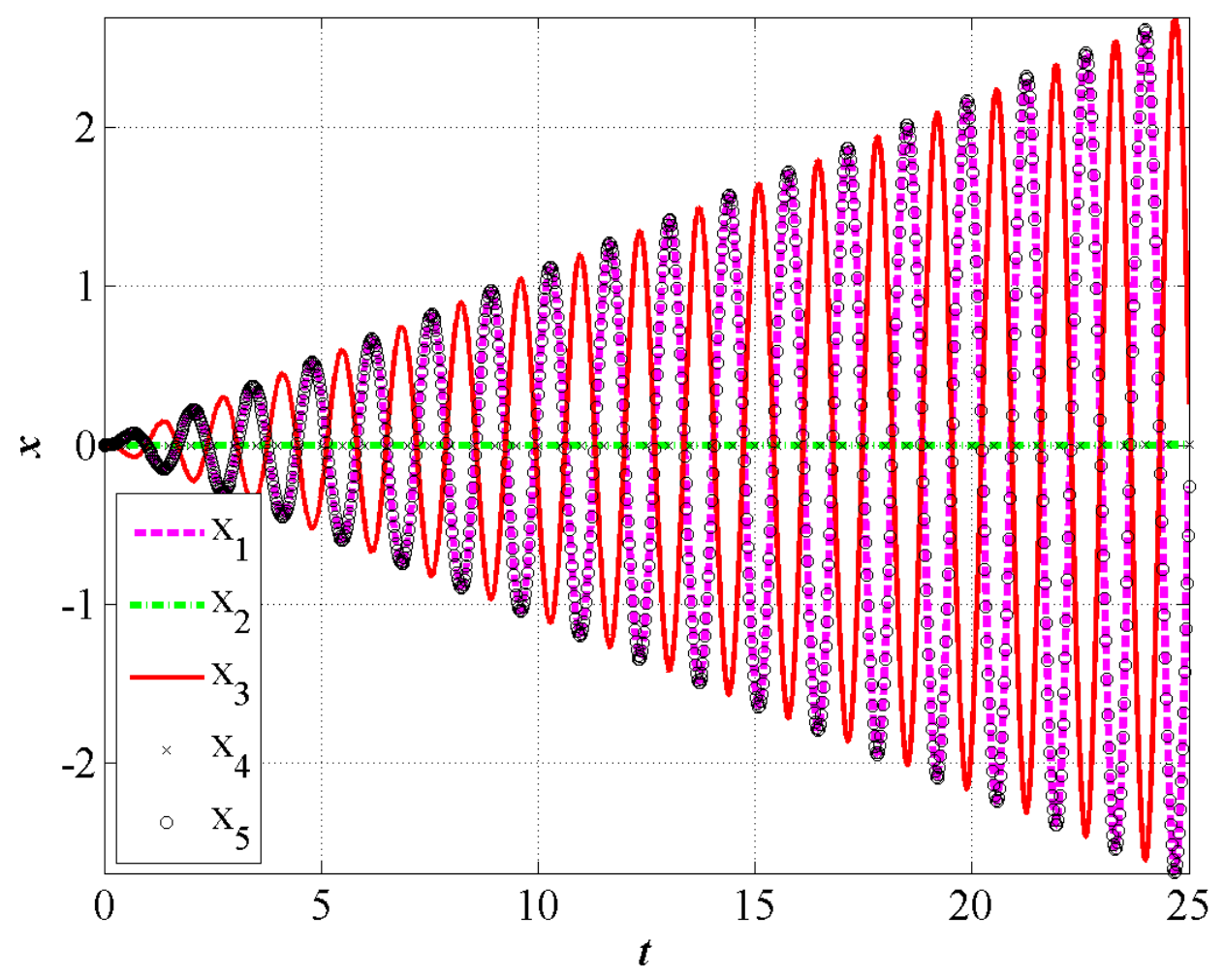

a) Motion for $\Omega=\Omega_{\mathrm{R}}$ 


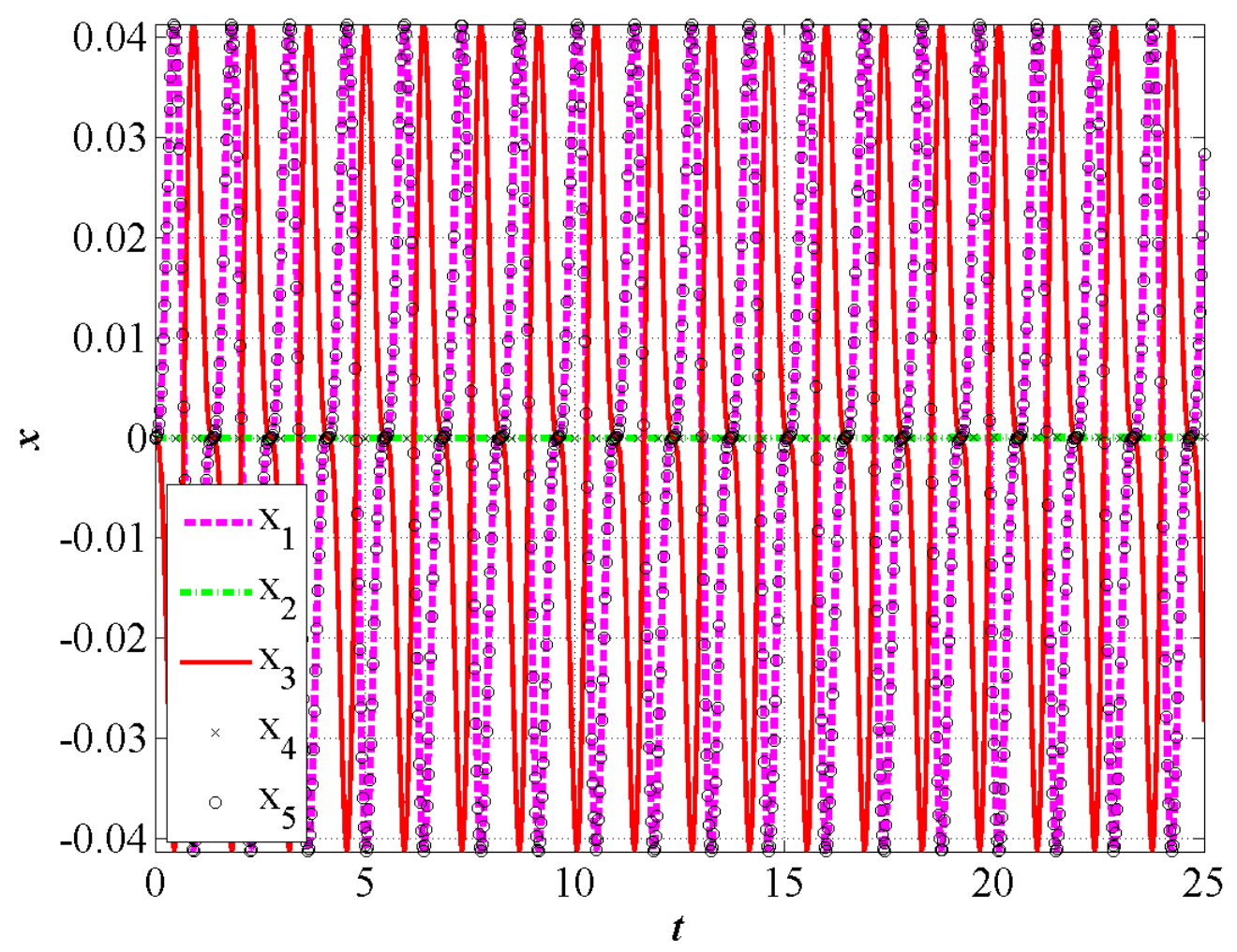

b) Motion for $\Omega=2 \Omega_{\mathrm{R}}$ 


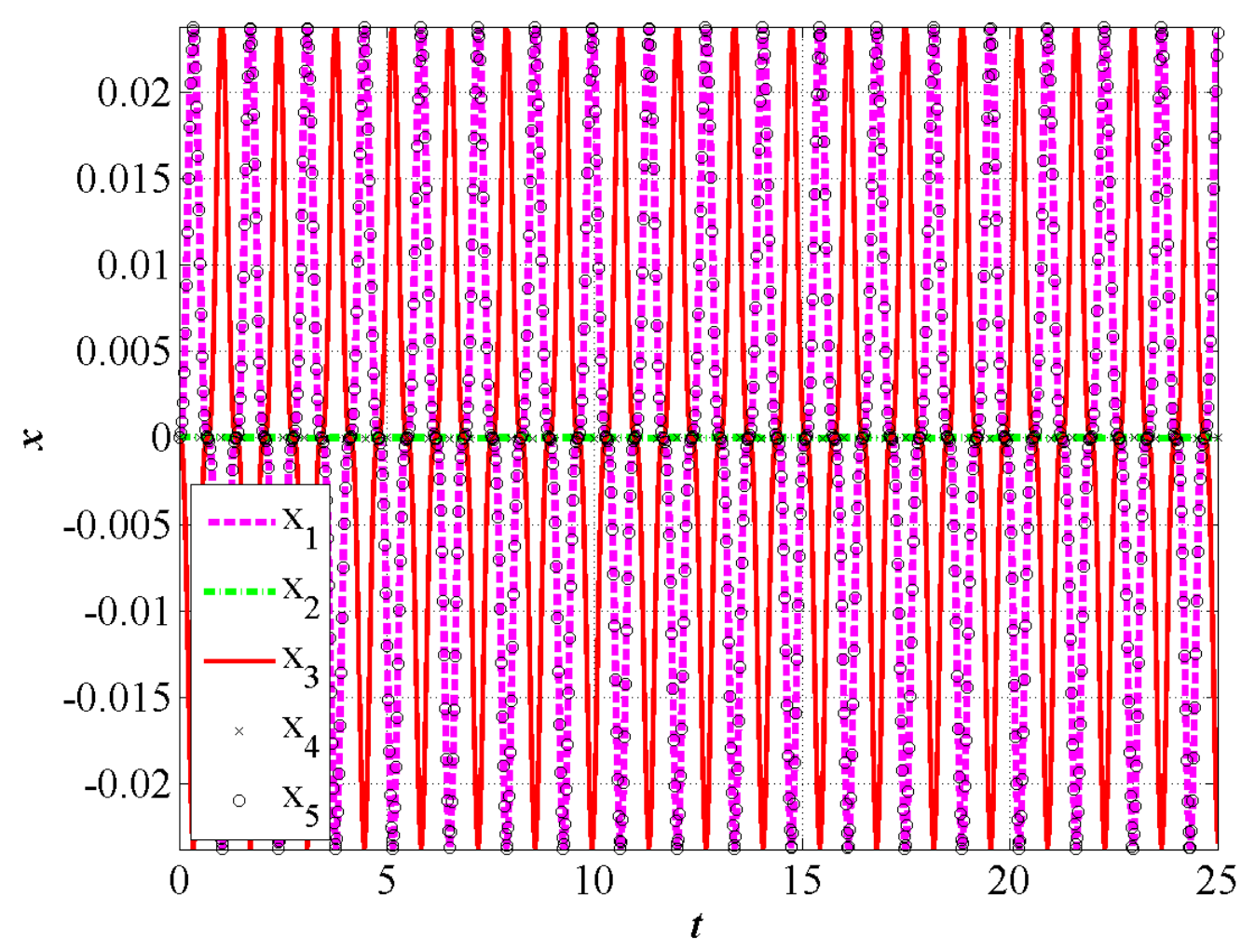

c) Motion for $\Omega=3 \Omega_{\mathrm{R}}$ 


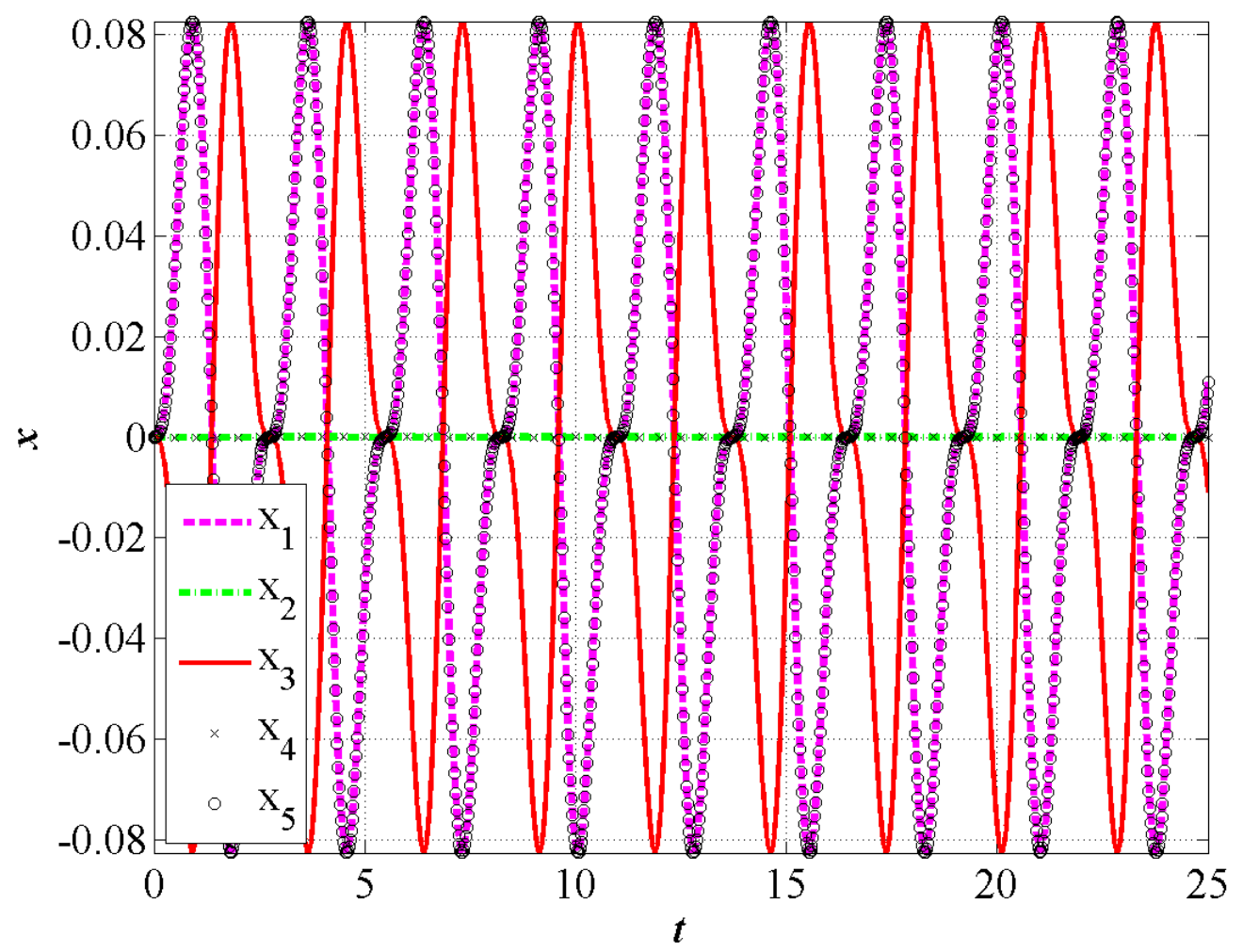

d) Motion for $\Omega=0.5 \Omega_{R}$

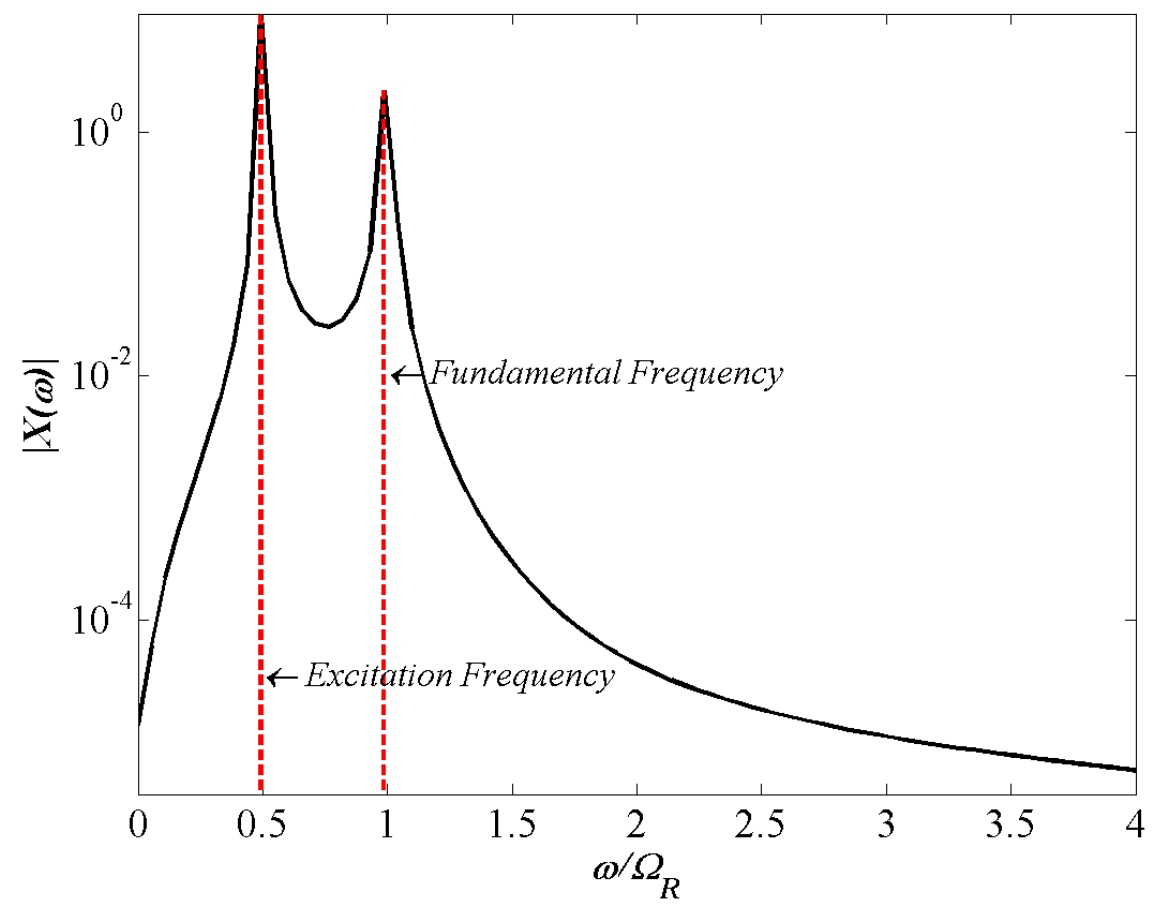

e) Spectrum for $\Omega=0.5 \Omega_{R}$ 


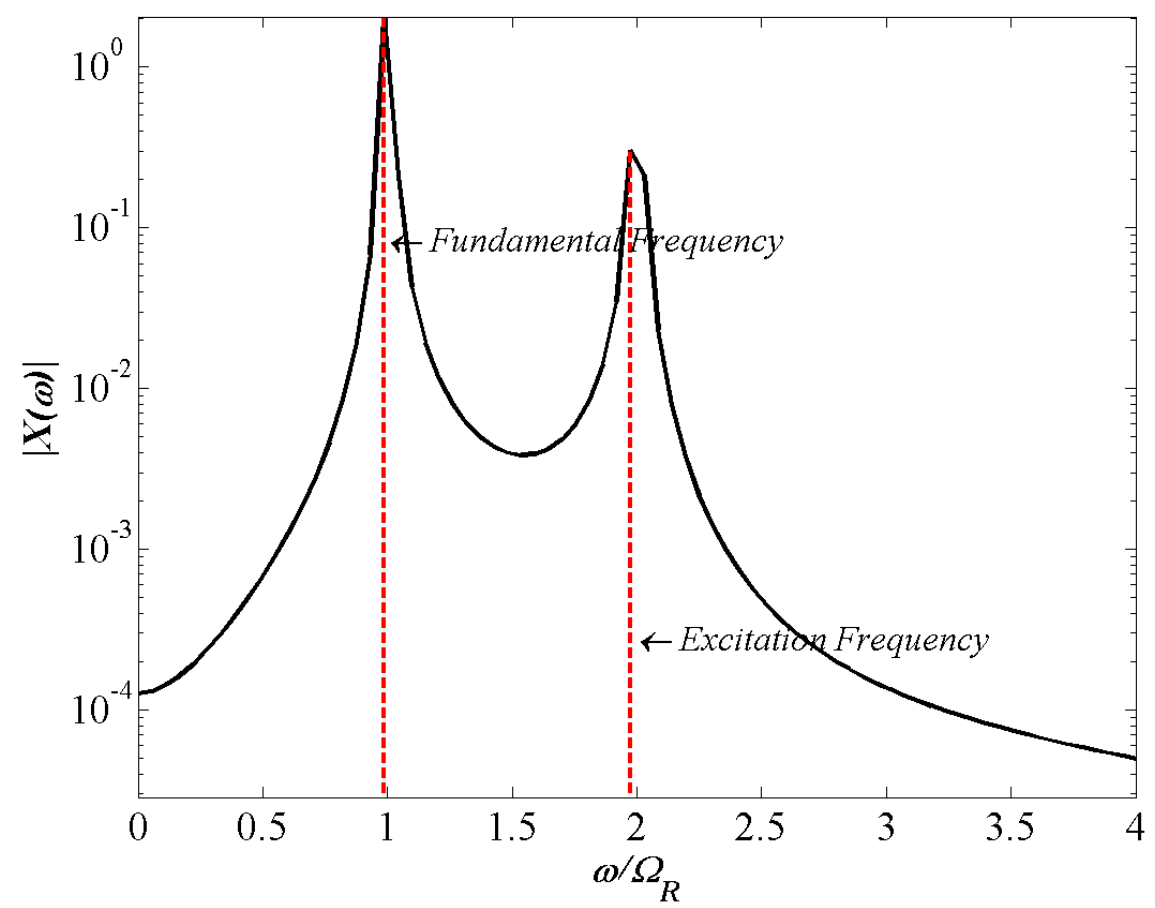

f) Spectrum for $\Omega=2 \Omega_{R}$

Figure 12. A five mass bilinear chain, which permits resonance decomposition into three single mass bilinear chains. The chain is excited by harmonic forces with the same frequency $\Omega$ applied in the directions specified by the eigenvector $(-1,0,1,0,-1), \Omega_{R}=4.5826, K=20, \omega_{+}=1$. 


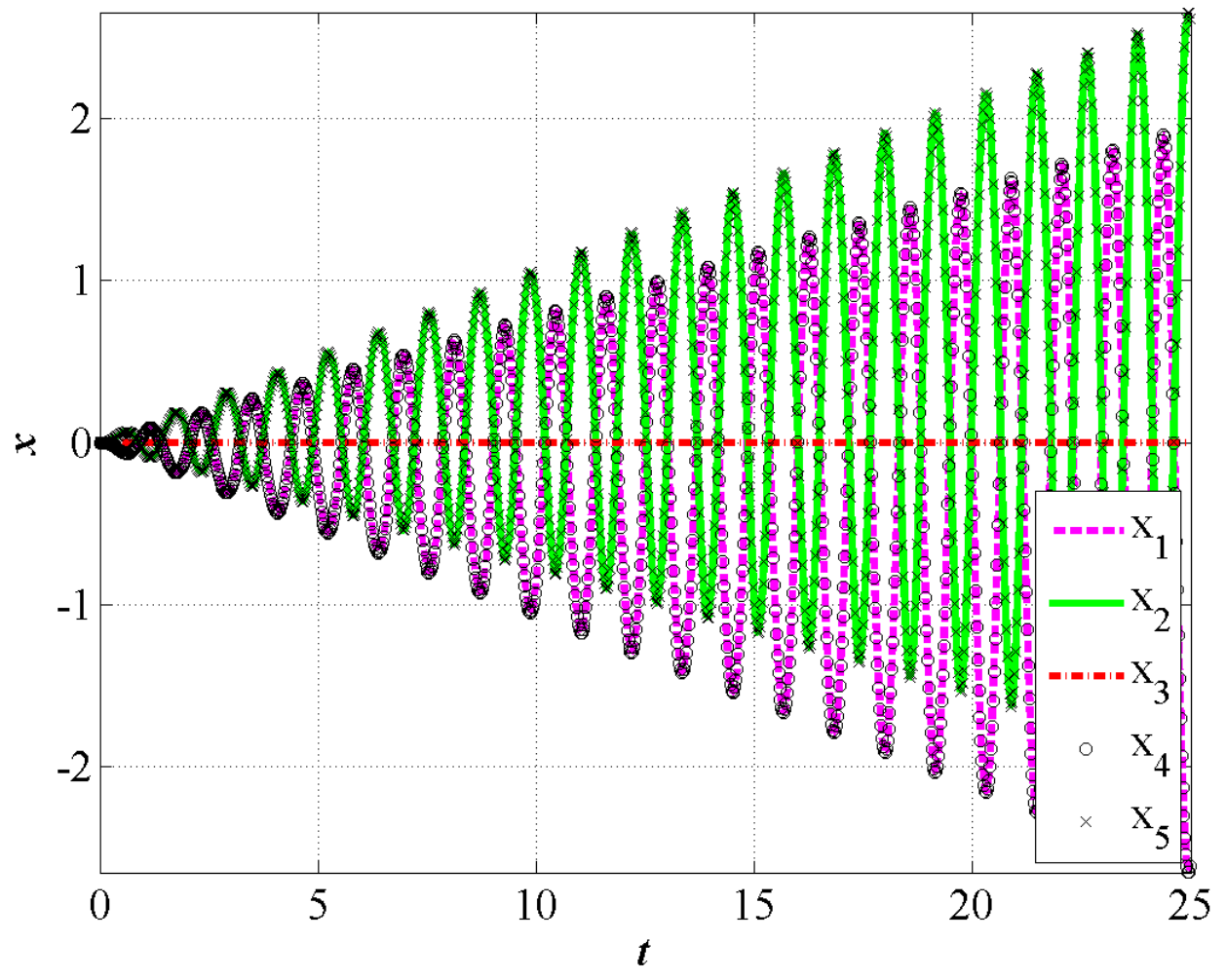

a) Motion for $\Omega=\Omega_{R}$ 


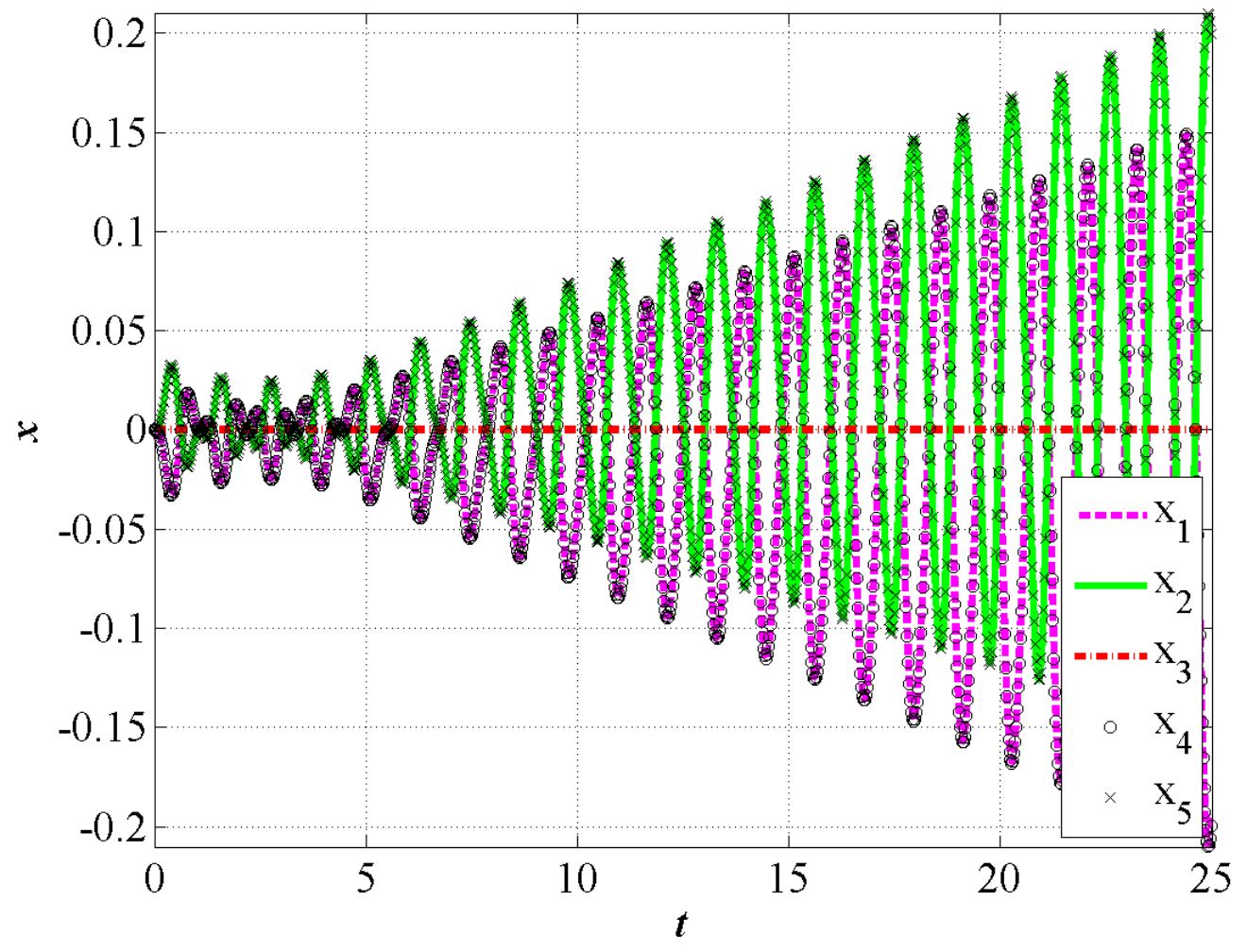

b) Motion for $\Omega=2 \Omega_{\mathrm{R}}$ 


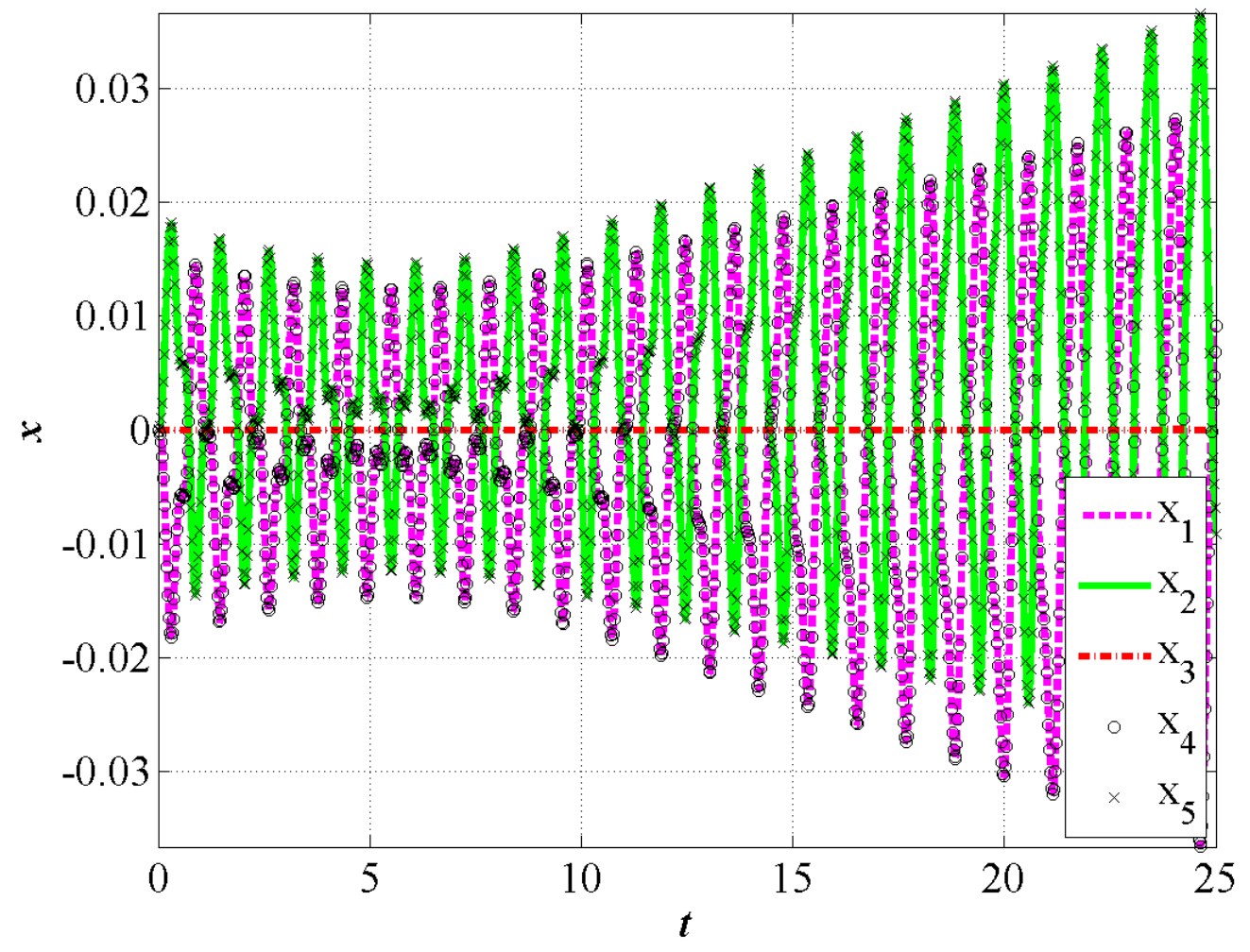

c) Motion for $\Omega=3 \Omega_{\mathrm{R}}$ 


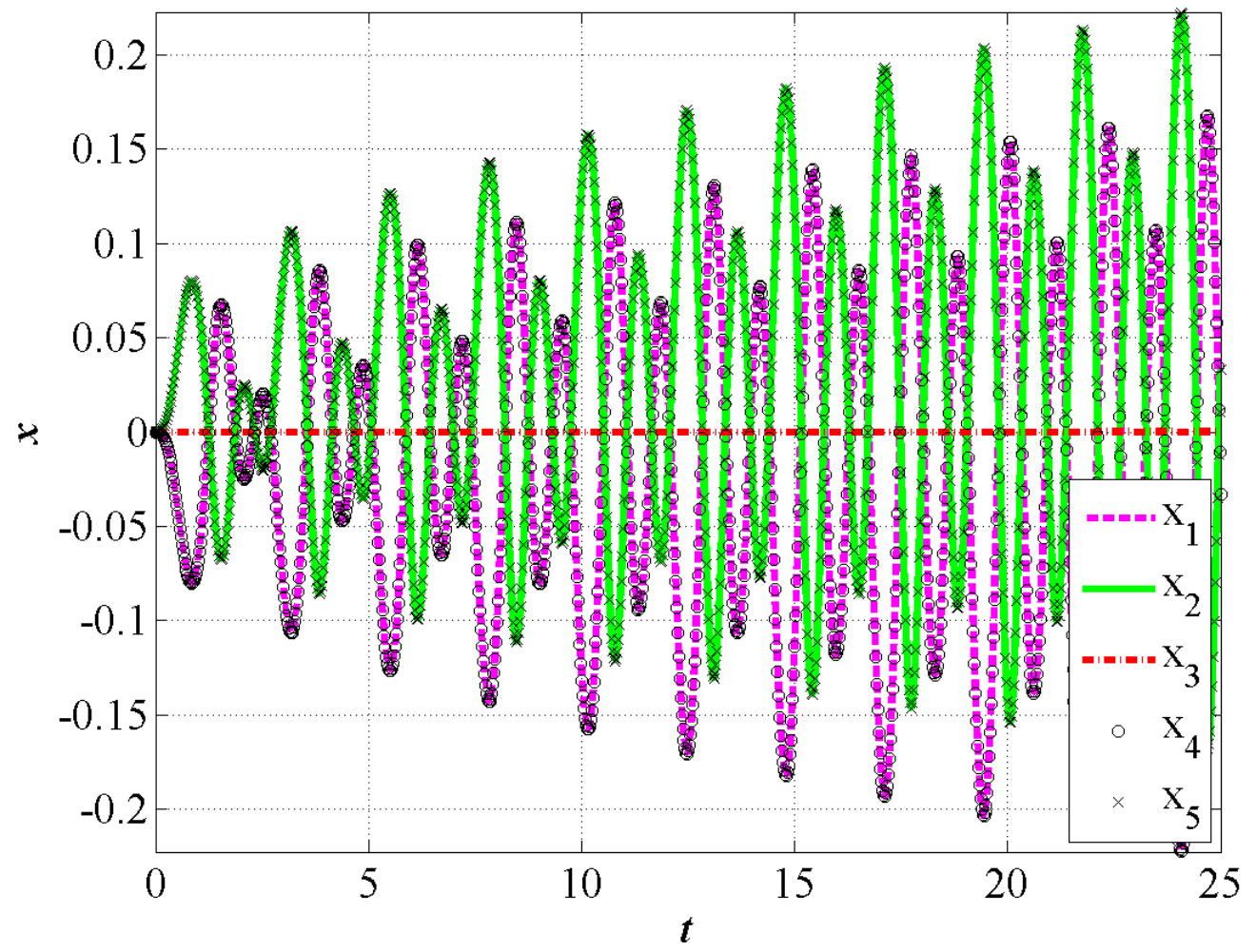

d) Motion for $\Omega=0.5 \Omega_{R}$

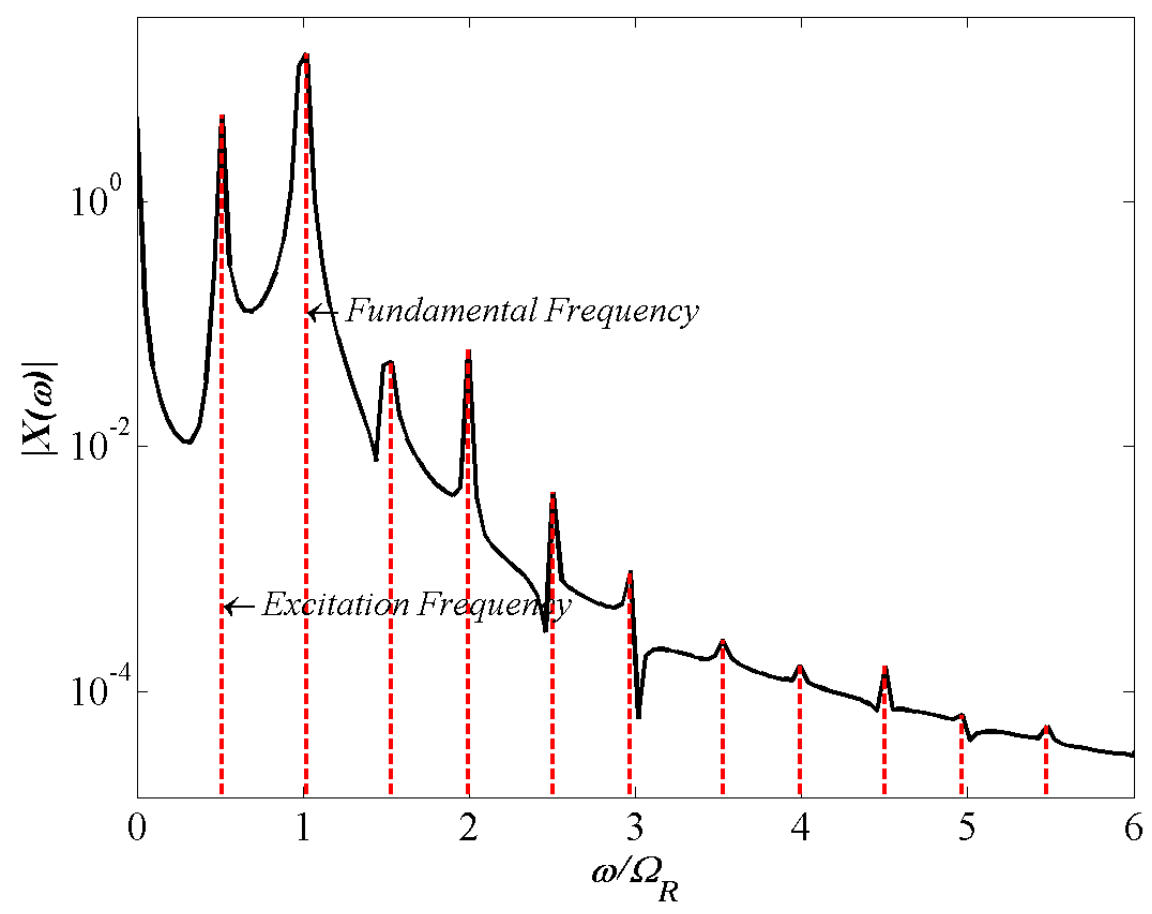

e) Spectrum for $\Omega=0.5 \Omega_{R}$ 


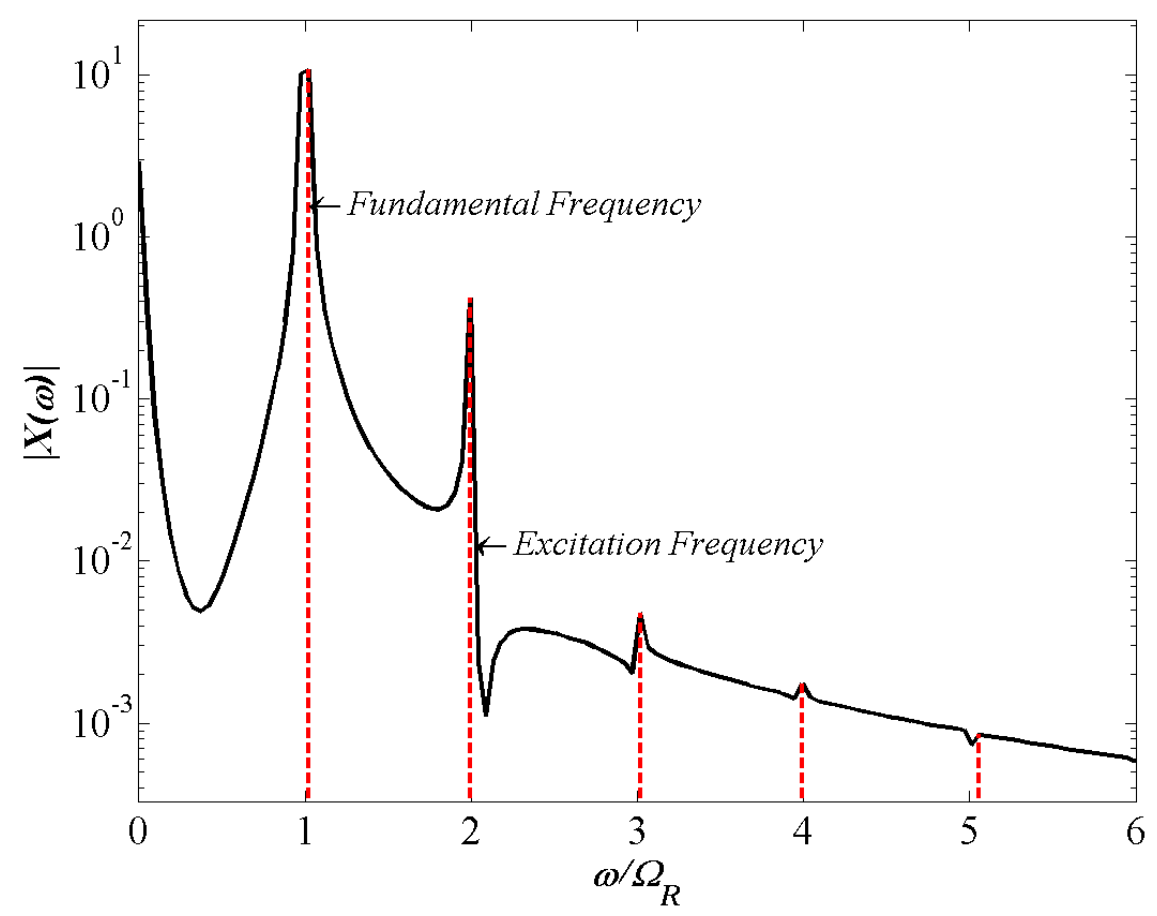

f) Spectrum for $\Omega=2 \Omega_{R}$

Figure 13. An eight mass bilinear chain, which permits resonance decomposition into three two mass bilinear chains. We show only trajectories $x_{1} \ldots x_{5}$ which are representative since $x_{1}=x_{4}=x_{7}$, $x_{2}=x_{5}=x_{8}, x_{3}=x_{6}=0$. The chain is excited by harmonic forces with the same frequency $\Omega$ applied in the directions specified by the eigenvector $(-1,1,0,-1,1,0,-1,1), \Omega_{R}=5.4146, K=20, \omega_{+}=1$.

It is seen that when the chain is decomposed into two mass bilinear chains it exhibits super and sub-harmonic resonances, i.e. at the frequencies multiple to the fundamental resonance frequency (super-harmonic resonances) and at least half resonant frequency (a sub-harmonic resonance), Figure 11. However, when the system is excited such that it decomposes into the single mass bilinear chains, it has only the fundamental resonance corresponding to the eigenfrequency of the one-mass oscillator, Figure 12. This is also seen in the spectra constructed for both cases, which reveal both super- and sub- harmonic picks in the case of decomposition into the two mass chains, Figure $11 \mathrm{e}, \mathrm{f}$ and neither of them for the single mass chain decomposition, Figure $12 \mathrm{e}, \mathrm{f}$. It is interesting to note that the sub-harmonic frequencies can only be seen on the spectrum constructed using the motion obtained at the half- resonant frequency, while the multiple resonance frequencies merely yield the spectrum with multiple frequencies. 
The last example is an eight mass chain, which is only decomposable into three two mass bilinear chains. Correspondingly it responds in a manner similar to a bilinear oscillator. Figure 13 shows the oscillations and frequency spectra of the chain excited in accordance with the eigenvector $(-1,1,0,-1,1,0,-1,1)$ with $\Omega_{R}=5.4146$. These plots clearly indicate the response of a bilinear chain with super- and sub-harmonic resonances on both motion and spectrum plots.

Figures 14,15 show trajectories $x_{i}$ vs. $x_{j}$ for the cases of four masses $(n=4)$ when no resonances are expected and five masses $(n=5)$ when two types of resonances exist. In the case of $n=4$, Figure 14, both frequencies, $\Omega_{R}=4.5826$ (the resonance frequency of the single mass bilinear chain) and $\Omega_{R}=5.4146$ (the resonance frequency of the two mass bilinear chain) are shown. The linear trajectories $x_{4}$ vs. $x_{1}$ (Figure $14 \mathrm{a}, \mathrm{c}$ ) and $x_{3}$ vs. $x_{2}$ (Figure $14 \mathrm{~b}, \mathrm{~d}$ ) reflect the way the chain was excited (see the excitation vectors in the caption). Other trajectories are chaotic reflecting the absence of the resonance. In the case of $n=5$, Figure 15, both these frequencies create resonances, which is reflected in all trajectories being linear. The horizontal lines reflect the stationary points, two for the case of $\Omega_{R}=4.5826$ when the chain gets decomposed into 3 single mass bilinear chains and one in the case of $\Omega_{R}=5.4146$ when the chain gets decomposed into 2 two mass bilinear chains. This pattern is observed for chains with other numbers of masses. 


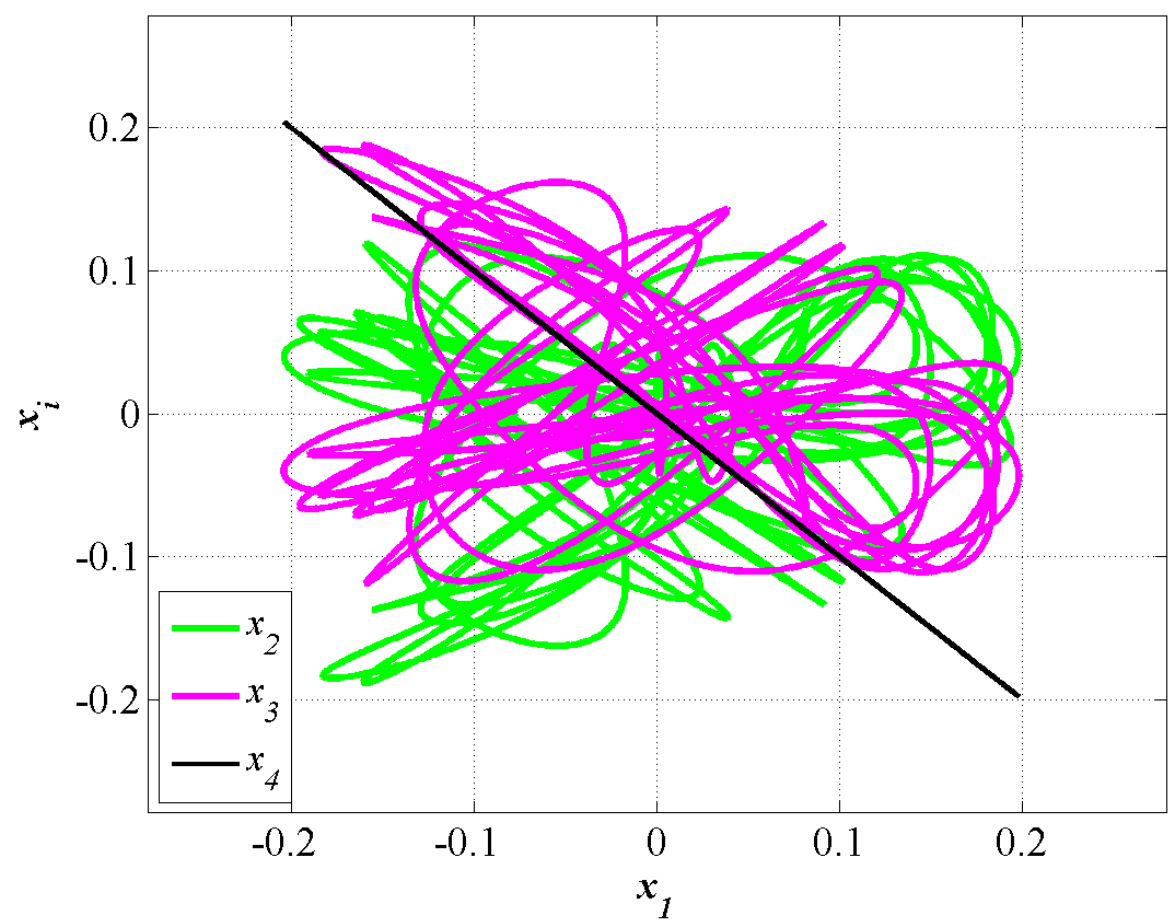

(a)

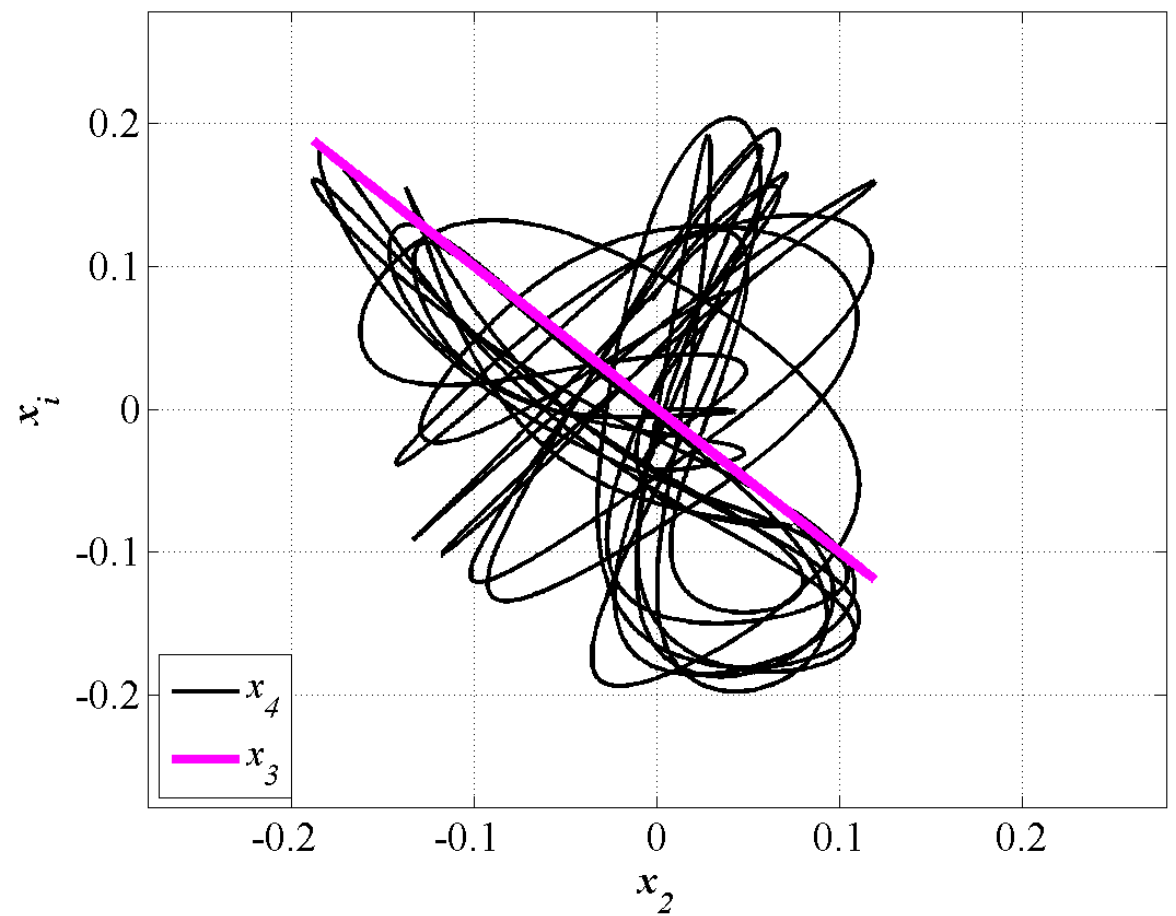

(b) 


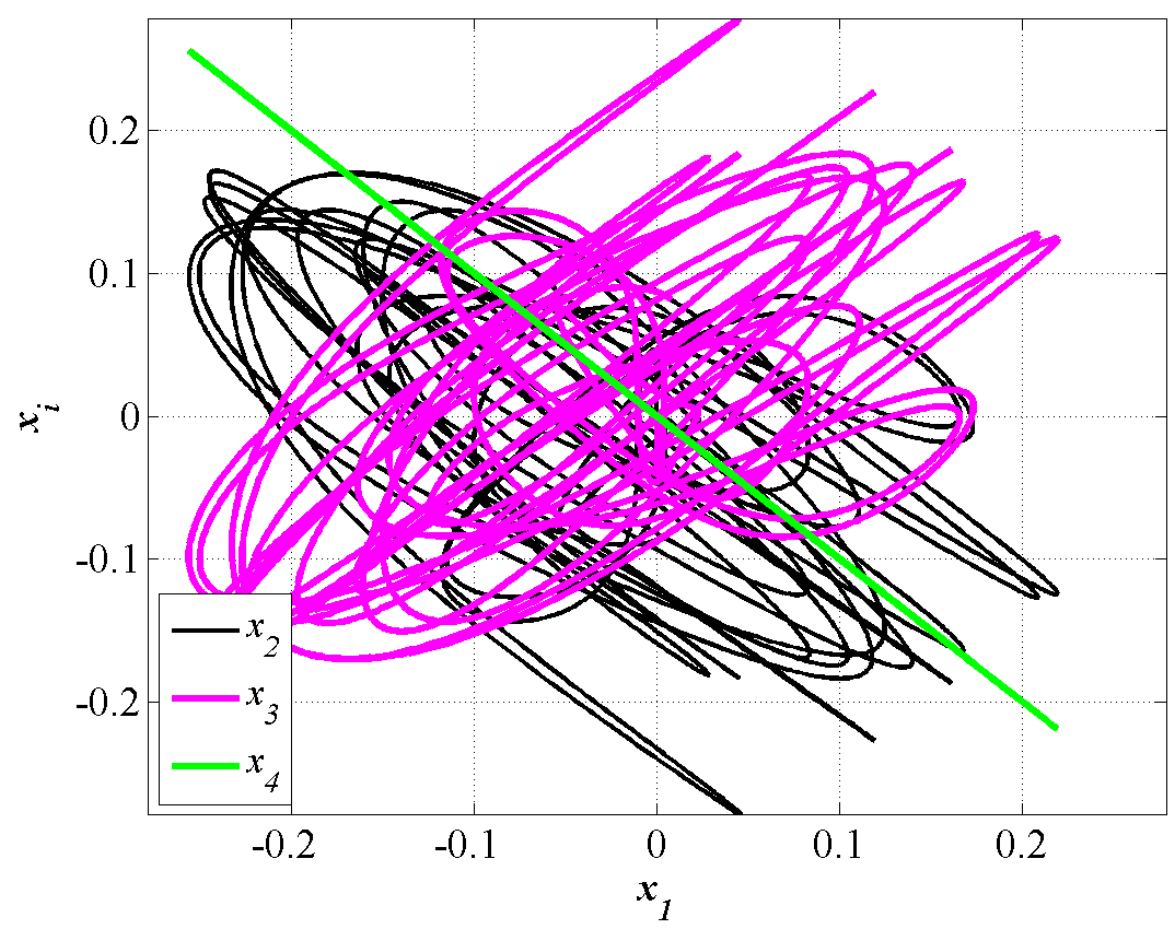

(c)

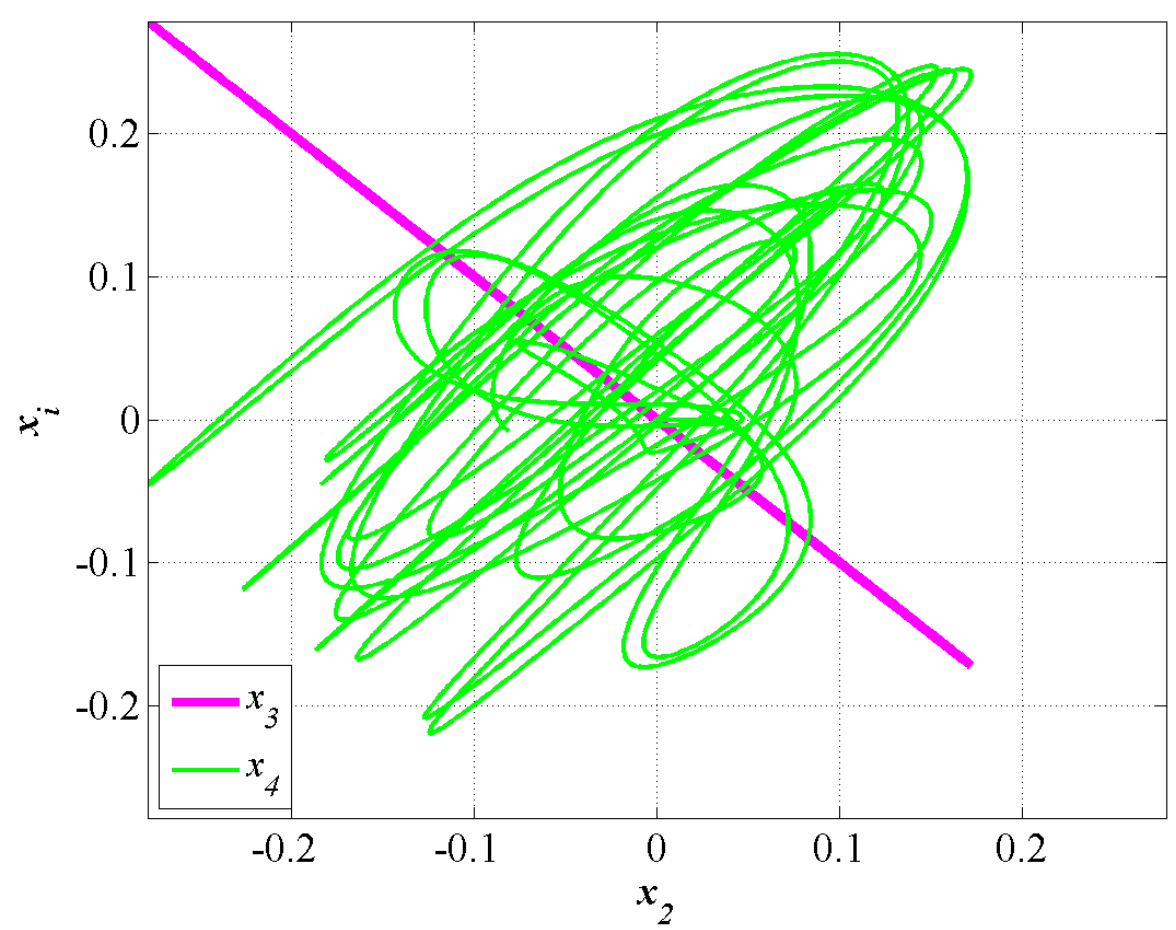

(d)

Figure 14: Trajectories of the systems of bilinear oscillators: 4 mass chains: a) $x_{i}$ vs. $x_{1}, n=4$, $\Omega_{R}=4.5826$, excitation vector $(0,-1,1,0)$; b) $x_{i}$ vs. $x_{2}, n=4, \Omega_{R}=4.5826$, excitation vector $(0,-1,1,0)$; c) $x_{i}$ vs. $x_{1}, n=4, \Omega_{R}=5.4146$, excitation vector $(0,-1,1,0)$; d) $x_{i}$ vs. $x_{2}, n=4$, $\Omega_{R}=5.4146$, excitation vector $(0,-1,1,0)$. 


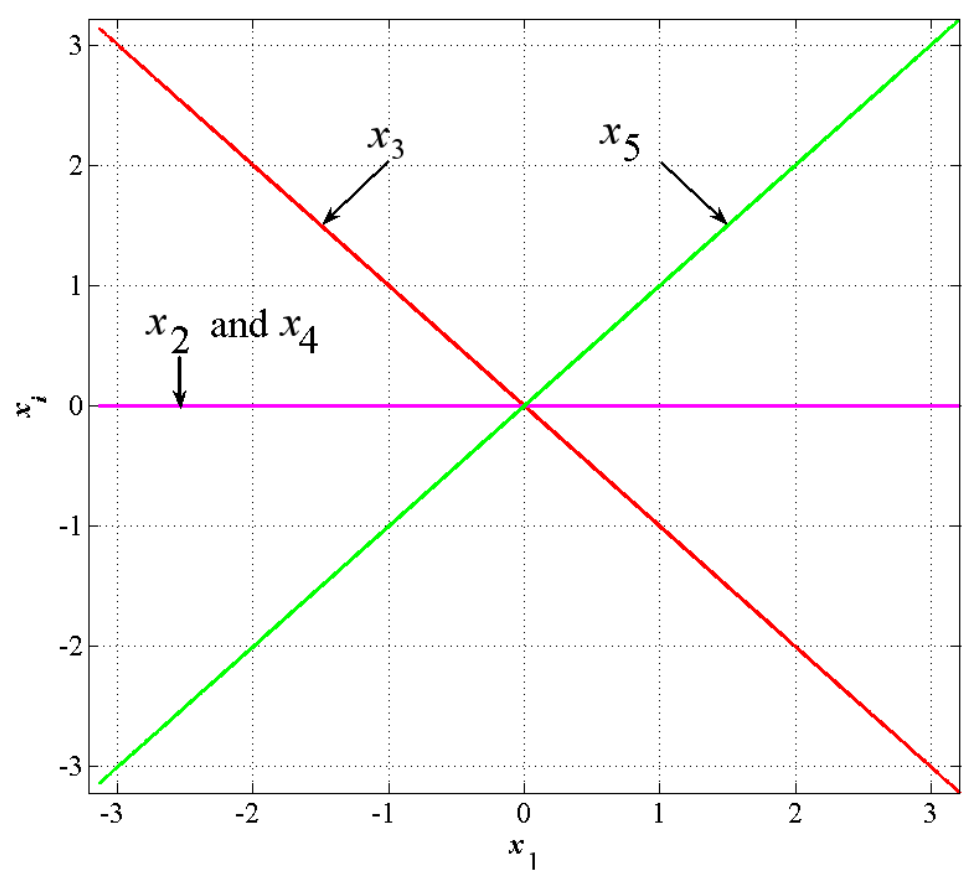

(a)

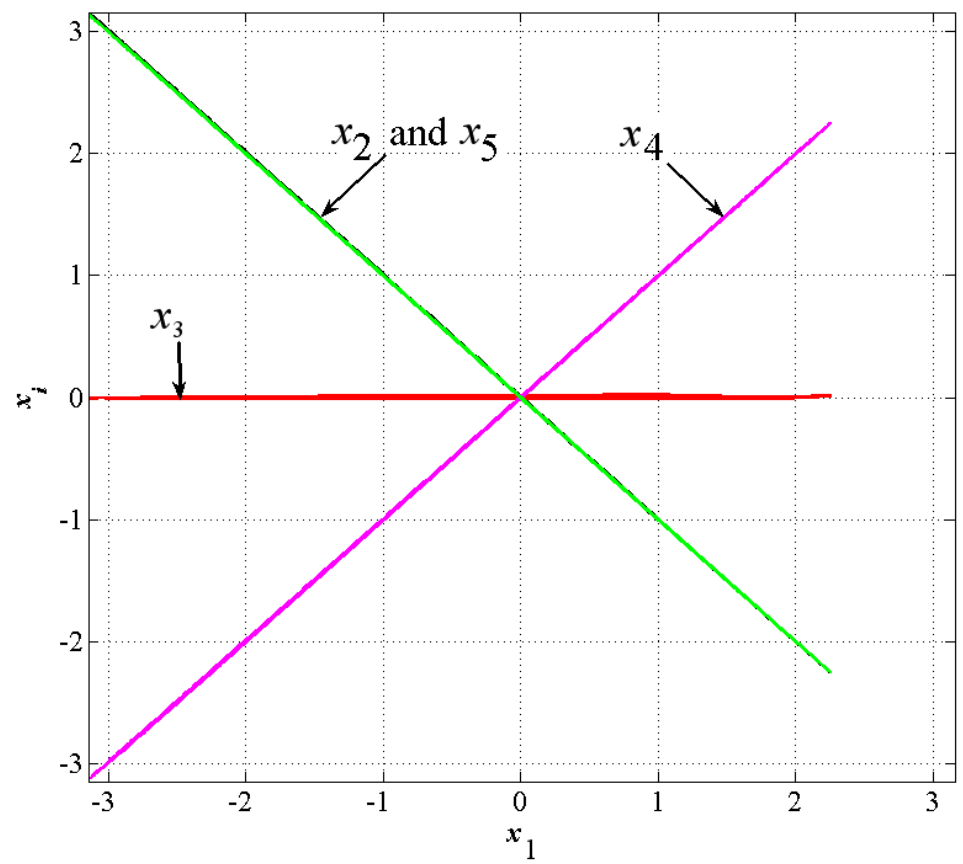

(b)

Figure 15: Trajectories of the systems of bilinear oscillators: 5 mass chains. Excitation vectors were chosen to coincide with the eigenvectors: (a) $n=5, \Omega_{R}=4.5826$ (decomposition into 3 single mass bilinear chains), excitation vector $(-1,0,1,0,-1)$; (b) $n=5, \Omega_{R}=5.4146$ (decomposition into 2 two mass bilinear chains), excitation vector $(-1,1,0,-1,1)$. 


\section{Discussion}

The absence of resonances or the presence of stationary points during resonances is an important factor in real vibrating chains, which places a limitation on the possible amplitudes of resonant modes. Indeed, the (piece-wise) linear vibrations in real structures correspond to the case of small amplitudes, as at higher amplitudes the vibrations become non-linear. The amplitudes should be small as compared to the length of the vibrating structure to satisfy the condition of infinitesimal deformations. The presence of stationary points breaks the vibrating structure into one- or twomass long chains. Then the amplitude of vibrations should be small compared to the length of these basic chains rather than the length of the total structure in order to keep (piece-wise) linearity. The increase in the amplitude above this restriction will make the system fully non-linear and the resonance will be lost. Thus the stationary points limit the possible amplitudes and increase the structural stability of the chain.

An obvious application of this concept is to fragmented solids, in particular to closed chains of blocks without bonding such as the mortarless arches. These and especially the topological interlocking structures where the special shapes of the blocks and their special arrangements provide the structural integrity without the need for keys, connectors or binder (e.g., [44-51]). In these structures the block interface is bilinear and all the blocks have the same mass and geometry. In this case the limitations on the possible amplitudes of resonances posed by the stationary points can provide a mechanism of amazing stability and longevity of these structures. Furthermore, the length (the number of blocks) becomes an important factor in controlling the structural stability. Obviously, the stability under imposed vibrations will be the highest if the number of blocks (stones) is such that no resonance is possible. The second best in terms of stability is the case when only one resonance arising from the decomposition into single mass bilinear chains is supported. The cases when the numbers of blocks are such that the chain is decomposed into two mass bilinear chains should be regarded as less stable since the two mass bilinear chains support superand sub-harmonic resonances. The least stable case is the case of the number of blocks supporting both types of decomposition, since then it can be excited on the resonance frequencies of the single mass chain and all super- and sub-harmonic resonance frequencies of the two mass chain. 


\section{Conclusions}

Bilinear oscillator is an important model that can represent strong nonlinearities observed in natural and engineering systems. While single bilinear oscillators were studied in depth, systems of multiple bilinear oscillators that model the behaviour of fragmented solids received much less attention. In order to fill this gap we investigated the simplest case - 1D symmetrical chains of bilinear oscillators with longitudinal movement. To this end we developed a new framework to analyse the resonances in closed symmetrical chains of bilinear oscillators (chains of bilinear oscillators with both ends fixed). The symmetry imposes specific patterns of resonances whereby the chain that achieved resonance is decomposed into a sequence of basic (either single mass or two mass) bilinear chains with stationary masses in between. This imposes restrictions on the lengths of the chains that support resonances in terms of the number of masses, $n$. Two types of lengths support the resonance: (1) odd numbers of masses $(n=2 m+1)$ leads to the chain decomposed into a series of single mass bilinear chains with stationary masses in between; (2) number of masses $n=3 m+2$, leads to the chain decomposed into a series of two mass bilinear chains. If length $n$ satisfies neither of these conditions, no resonance is possible. Alternatively, if the length satisfies both conditions (such as chains of lengths $5,11,17$,...masses), both types of resonances are accessible.

The resonance modes of a chain coincide with the eigenmodes of the basic chains. It is important to emphasise that the resonances in the whole chain of bilinear oscillators force some masses to become stationary (stationary points). The presence of stationary points is an important feature of the symmetric systems of bilinear oscillators. These stationary points separate the basic oscillators. Thus, the resonance decomposes the whole chain into a number of the basic chains, either single mass or two mass, but not a combination thereof.

The resonance frequency of the chain is inherited from the type of the basic chains the chain is decomposed into. Furthermore, the systems decomposable into two mass bilinear chains also possess super- and sub-harmonic resonances, while the chains decomposable into the single mass chains do not have super-harmonic and sub-harmonic resonances and behave like a linear 
oscillator. The chains that do not allow decomposition into the basic chains do not have resonances.

\section{Acknowledgements}

The authors acknowledge the financial support from the Australian Research Council through the Discovery Grant DP0988449.

\section{References}

1. M. Wiercigroch, Chaotic Vibration of a Simple Model of the Machine Tool-Cutting Process System, Journal of Vibration and Acoustics 119 (1997) 468-475.

2. M.A. Davies, B. Balachandran, Impact dynamics in milling of thin-walled structures. Nonlinear Dynamics 22 (2000) 375-392.

3. A.V. Dyskin, E. Pasternak, I. Shufrin, Vertical vibrations and detachments of drill bits in rotary drilling. $7^{\text {th }}$ Australasian Congress on Applied Mechanics, ACAM 7, 9-12 December 2012, Adelaide, Australia. Paper 115 (2012).

4. J.M.T. Thomson, Complex dynamics of compliant off-shore structures. Proc. Roy. Soc. London A, 387 (1983) 407-427.

5. J.M.T. Thompson, A.R. Bokaian, R. Ghaffari, Subharmonic resonances and chaotic motions of a bilinear oscillator. IMA Journal of Applied Mathematics 31 (1983) 207-234.

6. M. Gerber, L. Engelbrecht, The bilinear oscillator: The response of an articulated mooring tower driven by irregular seas. Ocean Engineering, 20 (1993) 113-133.

7. A. Raghothama, S. Narayanan, Bifurcation and chaos of an articulated loading platform with piecewise non-linear stiffness using the incremental harmonic balance method, Ocean Engineering 27 (2000) 1087-110.

8. M.S.T. De Freitas, R.L. Viana, Multistability, basin boundary structure, and chaotic behavior in a suspension bridge model. Internat. J. Bifurcation and Chaos 14 (2004) 927-950.

9. S.H. Doole, S.J. Hogan, A piecewise linear suspension bridge model: Nonlinear dynamics and orbit continuation, Dyn. Stab. Syst. 11 (1996), 19-47.

10. V.A., Lazaryan, L.A. Manashkin, A.V. Ryzhov, Starting one-dimensional mechanical systems with pretensioned shock absorbers. Prikladnaya Mechanica, 5 (1968) 64-70.

11. L. Adler, Double elasticity in drill cores under flexure. Int. J. Rock Mech. Min. Sci. Geomech. Abstr. 7 (1970) 357-370.

12. Y.C. Chu, H.H. Shen, Analysis of forced bilinear oscillators and the application to cracked beam dynamics, AIAA Journal 10 (1992) 2512-2519. 
13. J.A. Brandon, O.N.L. Abraham, Counter-intuitive quasi-periodic motion in the autonomous vibration of cracked Timoshenko beams. J. Sound and Vibration 185 (1995) 415-430.

14. M.B. Prime, D.W. Shevitz, Linear and nonlinear methods for detecting cracks in beams, in: Proc. 14th International Modal Analysis Conference, Dearborn, Michigan, pp., 1996, 14371443.

15. M. Chati, R. Rand, S. Mukherjee, Modal analysis of a cracked beam, Journal of Sound and Vibrations 207 (1997) 249-270.

16. A. Rivola, P.R. White, Bispectral analysis of the bilinear oscillator with application to the detection of fatigue cracks. J. Sound and Vibrations 216 (1998) 889-910.

17. T.G. Chondros, A.D. Dimarogonas, J. Yao, Vibration of a beam with a breathing crack. J. Sound and Vibration 239 (2001) 57-67.

18. U. Andreaus, P. Casini, F. Vestroni, Non-linear dynamics of a cracked cantilever beam under harmonic excitation, International Journal of Non-Linear Mechanics 42 (2007) 566-575.

19. Z.K. Peng, Z.Q. Lang, F.L. Chu, Numerical analysis of cracked beams using nonlinear output frequency response functions. Computers \& Structures 86 (2008) 1809-1818.

20. F. Vestroni, A. Luongo, A. Paolone, A perturbation method for evaluating nonlinear normal modes of a piecewise linear two-degrees-of-freedom system, Nonlinear Dynamics 54 (2008) 379-393.

21. V. Lyakhovsky, Y. Hamiel, P. Ampuero, Y. Ben-Zion, Nonlinear damage rheology and wave resonance in rocks. Int. J. Geophys. 178 (2009) 910-920.

22. V.R. Hiwarkar, V.I. Babitsky, V.V. Silberschmidt, Crack as modulator, detector and amplifier in structural health monitoring, Journal of Sound and Vibrations 331 (2012) 3587-3598.

23. M.C. Teich, S.E. Keilson, S.M. Hanna, Models of non-linear vibration. II. Oscillator with bilinear stiffness, Acta Otolaryngol. (Stockh), Suppl. 467 (1989) 249-256.

24. S. Natsiavas, Dynamics of multiple-degree-of-freedom oscillators with colliding components, Journal of Sound and Vibration 165 (1993) 439-453.

25. G.S. Whiston, The vibro-impact response of a harmonically excited and preloaded onedimensional linear oscillator, Journal of Sound and Vibration, 115 (1987) 303-319.

26. A.B. Nordmark, Non-periodic motion caused by grazing incidence in impact oscillators, Journal of Sound and Vibration, 145(2) (1991) 279-297.

27. A.C.J. Luo, L. Chen, Periodic motions and grazing in a harmonically forced, piecewise, linear oscillator with impacts, Chaos, Solitons and Fractals, 24 (2005) 567-578.

28. A.C.J. Luo, M.T. Patel, Bifurcation and stability of periodic motions in a periodically forced oscillator with multiple discontinuities, Journal of Computational and Nonlinear Dynamics 4 (2009) 011011.

29. A.V. Dyskin, E. Pasternak, E. Pelinovsky, Modelling resonances in topological interlocking structures, in: F. Albermani, B. Daniel, J. Griffiths, D. Hargreaves, P. Meehan, A. Tan, M. Veidt (Eds) ACAM2007, Proc. 5th Australasian Congress on Applied Mechanics, 10-12 December 2007, Brisbane, Australia 2, 2007, pp. 408-413. 
30. M. Carlesso, A. Molotnikov, T. Krause, K. Tushtev, S. Kroll, K. Rezwan, Y. Estrin, Enhancement of sound absorption properties using topologically interlocked elements, Scripta Materialia, 66 (2012) 483-486.

31. S.W. Shaw, P.J. Holmes, A periodically forced piecewise linear oscillator. J. Sound and Vibration 90 (1983) 129-155.

32. C. W. Wong, W. -S. Zhang, S. L. Lau, Periodic forced vibration of unsymmetrical piecewiselinear systems by incremental Harmonic Balance method, Journal of Sound and Vibration 149(1) (1991) 91-05.

33. S.L. Lau, W.-S. Zhang, Nonlinear vibrations of piecewise-linear systems by incremental harmonic balance method, Journal of Applied Mechanics 59 (1992) 153-160.

34. J.C.Ji, A.Y.T. Leung, "Periodic and chaotic motions of a harmonically forced piecewise linear system”, International Journal of Mechanical Sciences, 46 (2004) 1807-1825.

35. A.V. Dyskin, E. Pasternak, E. Pelinovsky, Periodic motions and resonances of impact oscillators, Journal of Sound and Vibrations, 331 (2012) 2856-2873.

36. I.Yu. Solodov, B.A. Korshak, Instability, chaos, and "memory" in acoustic-wave-crack interaction. Physical Review Letters 88(2002) 041303.

37. I.Yu. Solodov, J. Wackerl, K. Pfleiderer, G. Busse, Nonlinear self-modulation and subharmonic acoustic spectroscopy for damage detection and location. Applied Physic Letters, 84 (2004) 5386-5388.

38. D.J. Wagg, S.R. Bishop, Dynamics of a two degree of freedom vibro-impact system with multiple motion limiting constraints. International Journal of Bifurcation and Chaos. 14 (2004) 119-140.

39. P. Casini, F. Vestroni, Characterization of bifurcating non-linear normal modes in piecewise linear mechanical systems, International Journal of Non-Linear Mechanics, 46 (2011), 142150.

40. O. Giannini, P. Casini, F. Vestroni, Experimental evidence of bifurcating nonlinear normal modes in piecewise linear systems, Nonlinear Dynamics, 63 (2011), 655-666.

41. V.N. Pilipchuk, Closed-form periodic solutions for piecewise-linear vibrating systems, Nonlinear Dynamics, 58 (2009) 169-178.

42. I. Shufrin, A.V. Dyskin, E. Pasternak, Stationary points created by resonances in a chain of bilinear oscillators. $7^{\text {th }}$ Australasian Congress on Applied Mechanics, ACAM 7, 9-12 December 2012, Adelaide, Australia. Paper 131 (2012).

43. M. Golubitsky, I. Stewart, Nonlinear dynamics of networks: the groupoid formalism. Bulletin (new series) of the American Mathematical Society, 43 (2006), 305-364.

44. Y. Estrin, A.V. Dyskin, A.J. Kanel-Belov, E. Pasternak, Materials with novel architectonics: assemblies of interlocked elements, IUTAM Symposium on Analytical and Computational Fracture Mechanics of Non-homogeneous Materials, (Karihaloo, B., Ed), Cardiff, UK, June 18-22, 2001, Kluwer Academic Publishers, 51-55 (2002). 
45. H.C. Khor, A.V. Dyskin, E. Pasternak, Y. Estrin, A.J. Kanel-Belov, Integrity and fracture of plate-like assemblies of topologically interlocked elements, Structural Integrity and Fracture (Dyskin, A.V., Hu X.Z. and Sahouryeh, E., Eds), Swets \& Zeitlinger, Lisse, 449-456 (2002).

46. A.V. Dyskin, Y. Estrin, E. Pasternak, H.C. Khor, A.J. Kanel-Belov, Fracture resistant structures based on topological interlocking with non-planar contacts, Advanced Engineering Materials 5 (2003), 116-119.

47. Y. Estrin, A.V. Dyskin, E. Pasternak, S. Schaare, S. Stanchits, A.J. Kanel-Belov, Negative stiffness of a layer with topologically interlocked elements, Scripta Materialia, 50 (2003), 291294.

48. A.V. Dyskin, Y. Estrin, A.J. Kanel-Belov, E. Pasternak, Interlocking properties of buckyballs, Physics Letters A, 319 (2003), 373-378.

49. S. Schaare, A.V. Dyskin, Y. Estrin, S. Arndt, E. Pasternak, A. Kanel-Belov, Point Loading of Assemblies of Interlocked Cube-Shaped Elements, Int. J. Engineering Science, 46 (2008), $1228-1238$.

50. A.J. Kanel-Belov, A.V. Dyskin, Y. Estrin, E., Pasternak, I.A. Ivanov, Interlocking of convex polyhedra: towards a geometric theory of fragmented solids, Moscow Mathematical Journal, 10 (2010), 337-342.

51. A.V. Dyskin, E. Pasternak, Y. Estrin, Mortaless structures based on topological interlocking, Frontiers of Structural and Civil Engineering, 6 (2012) 188-197. 